\title{
Diamond alpha Hardy-Copson type dynamic inequalities
}

\author{
Zeynep Kayar*1 (D), Billur Kaymakçalan² \\ ${ }^{1}$ Van Yüzüncü Yal University, Department of Mathematics, 65080, Van, Turkey \\ ${ }^{2}$ Çankaya University, Department of Mathematics, 06810, Ankara, Turkey
}

\begin{abstract}
In this paper two kinds of dynamic Hardy-Copson type inequalities are derived via diamond alpha integrals. The first kind consists of twelve new integral inequalities which can be considered as mixed type in the sense that these inequalities contain delta, nabla and diamond alpha integrals together. The second kind involves another twelve new inequalities, which are composed of only diamond alpha integrals, unifying delta and nabla Hardy-Copson type inequalities. Our approach is quite new due to the fact that it uses time scale calculus rather than algebra. Therefore both kinds of our results unify some of the known delta and nabla Hardy-Copson type inequalities into one diamond alpha Hardy-Copson type inequalities and offer new Hardy-Copson type inequalities even for the special cases.
\end{abstract}

Mathematics Subject Classification (2020). 34N05, 26D10, 26E70

Keywords. diamond alpha calculus, Hardy's inequality, Copson's inequality

\section{Introduction}

The theory of inequalities containing series or integrals has been given a great importance due to their effective usage in differential equations and their applications after the appearance of the celebrated discrete and continuous inequalities of Hardy. In 1920, when Hardy [28] tried to find a simple and elementary proof of Hilbert's inequality [42]

$$
\sum_{n=1}^{\infty} \sum_{m=1}^{\infty} \frac{a_{m} c_{n}}{m+n} \leq \pi\left(\sum_{m=1}^{\infty} a_{m}^{2}\right)^{1 / 2}\left(\sum_{n=1}^{\infty} c_{n}^{2}\right)^{1 / 2}
$$

where $a_{m}, c_{n} \geq 0$ and $\sum_{m=1}^{\infty} a_{m}^{2}$ and $\sum_{n=1}^{\infty} c_{n}^{2}$ are convergent, he showed the following pioneering discrete inequality

$$
\sum_{j=1}^{\infty}\left(\frac{1}{j} \sum_{i=1}^{m} c(i)\right)^{\zeta} \leq\left(\frac{\zeta}{\zeta-1}\right)^{\zeta} \sum_{j=1}^{\infty} c^{\zeta}(j), \quad c(j) \geq 0, \zeta>1
$$

\footnotetext{
*Corresponding Author.

Email addresses: zeynepkayar@yyu.edu.tr (Z. Kayar), billurkaymakcalan@gmail.com (B. Kaymakçalan) Received: 26.04.2021; Accepted: 05.08.2021
} 
and pioneering continuous inequality for a nonnegative function $\psi$ and for a real constant $\zeta>1$, as

$$
\int_{0}^{\infty}\left(\frac{1}{t} \int_{0}^{t} \psi(s) d s\right)^{\zeta} d t \leq\left(\frac{\zeta}{\zeta-1}\right)^{\zeta} \int_{0}^{\infty} \psi^{\zeta}(t) d t
$$

where $\int_{0}^{\infty} \psi^{\zeta}(t) d t<\infty$. In fact, Hardy only stated inequality (1.2) in [28] but did not prove it. After that in 1925, the proof of inequality (1.2), which depends on the calculus of variations, was shown by Hardy in [29].

The constant $\left(\frac{\zeta}{\zeta-1}\right)^{\zeta}$ that appears in the above inequalities also has been found as the best possible one, since if it is replaced by a smaller constant then inequalities (1.1) and (1.2) are not fulfilled anymore for the involved sequences and functions, respectively.

Then Hardy et al. [30, Theroem 330] developed inequality (1.2) and derived the following integral inequality for a nonnegative function $\psi$ as

$$
\int_{0}^{\infty} \frac{\Psi^{\zeta}(t)}{t^{\theta}} d t \leq\left|\frac{\zeta}{\theta-1}\right|^{\zeta} \int_{0}^{\infty} \frac{\psi^{\zeta}(t)}{t^{\theta-\zeta}} d t, \quad \zeta>1
$$

where if $\theta>1$, then $\Psi(t)=\int_{0}^{t} \psi(s) d s$ and if $\theta<1$, then $\Psi(t)=\int_{t}^{\infty} \psi(s) d s$.

The exhibition of the results containing the improvements, generalizations and applications of the discrete and continuous Hardy inequalities (1.1)-(1.3) can be found in the books $[9,30,42,43,49]$ and references therein. In particular the discrete case was investigated in $[11,19,20,24,44-47]$ while the continuous Hardy inequalities were analyzed in $[10,21,31,52,54,55]$.

If the arithmetic mean of a sequence $c(j)$ is replaced by its weighted arithmetic mean, then two discrete inequalities, one of which refines discrete inequality (1.1) while the other one is a novel inequality, have been established by Copson [20, Theorem 1.1, Theorem 2.1] as follows.

Let $c(j)$ and $z(j)$ be nonnegative sequences for $j=1,2, \cdots$. If $\zeta>1, \theta>1$, then

$$
\sum_{j=1}^{\infty} \frac{z(j)}{[\bar{G}(j)]^{\theta}}\left(\sum_{i=1}^{j} c(i) z(i)\right)^{\zeta} \leq\left(\frac{\zeta}{\theta-1}\right)^{\zeta} \sum_{j=1}^{\infty} z(j)[\bar{G}(j)]^{\zeta-\theta} c^{\zeta}(j)
$$

and if $0 \leq \theta<1<\zeta$, then

$$
\sum_{j=1}^{\infty} \frac{z(j)}{[\bar{G}(j)]^{\theta}}\left(\sum_{i=j}^{\infty} c(i) z(i)\right)^{\zeta} \leq\left(\frac{\zeta}{1-\theta}\right)^{\zeta} \sum_{j=1}^{\infty} z(j)[\bar{G}(j)]^{\zeta-\theta} c^{\zeta}(j)
$$

where $\bar{G}(j)=\sum_{i=1}^{j} z(i)$.

It is worth mentioning the concept introduced by Leindler in [46] for the inequalities involving series. Let the heads and the tails of a sequence $x$ be defined by

$$
x_{1, m}=\sum_{j=1}^{m} x(j) \text { and } x_{m, \infty}=\sum_{j=m}^{\infty} x(j),
$$

repectively. By this definition and by using the tails of the sequence $z$, Bennett [11, Corollary 3-Corollary 6] (see also Leindler [45, Proposition 1-Proposition 4]) obtained two more inequalities in addition to inequalities (1.4) and (1.5) as follows: 
Let the sequences $c(j)$ and $z(j)$ be nonnegative for $j=1,2, \cdots$ and $\sum_{i=1}^{\infty} z(i)<\infty$. Let us define the heads and the tails of the sequence $z$ as

$$
G(j)=\sum_{i=j}^{\infty} z(i) \text { and } \bar{G}(j)=\sum_{i=1}^{j} z(i),
$$

respectively. If $1<\theta \leq \zeta$, then

$$
\sum_{j=1}^{\infty} \frac{z(j)}{[G(j)]^{\theta}}\left(\sum_{i=j}^{\infty} c(i) z(i)\right)^{\zeta} \leq\left(\frac{\zeta}{\theta-1}\right)^{\zeta} \sum_{j=1}^{\infty} z(j)[G(j)]^{\zeta-\theta} c^{\zeta}(j),
$$

and

$$
\sum_{j=1}^{\infty} \frac{z(j)}{[\bar{G}(j)]^{\theta}}\left(\sum_{i=1}^{j} c(i) z(i)\right)^{\zeta} \leq\left(\frac{\zeta}{\theta-1}\right)^{\zeta} \sum_{j=1}^{\infty} z(j)[\bar{G}(j)]^{\zeta-\theta} c^{\zeta}(j) .
$$

If $0 \leq \theta<1<\zeta$, then

$$
\sum_{j=1}^{\infty} \frac{z(j)}{[G(j)]^{\theta}}\left(\sum_{i=1}^{j} c(i) z(i)\right)^{\zeta} \leq\left(\frac{\zeta}{1-\theta}\right)^{\zeta} \sum_{j=1}^{\infty} z(j)[G(j)]^{\zeta-\theta} c^{\zeta}(j),
$$

and

$$
\sum_{j=1}^{\infty} \frac{z(j)}{[\bar{G}(j)]^{\theta}}\left(\sum_{i=j}^{\infty} c(i) z(i)\right)^{\zeta} \leq\left(\frac{\zeta}{1-\theta}\right)^{\zeta} \sum_{j=1}^{\infty} z(j)[\bar{G}(j)]^{\zeta-\theta} c^{\zeta}(j) .
$$

Therefore by using this terminology, Bennett [11] (or Leindler [45]) treated the following four cases:

\begin{tabular}{|l|c|c|c|}
\hline Corollary 3 (or Proposition 1) & $1<\theta \leq \zeta$ & heads of $z$ & heads of $c z$ \\
\hline Corollary 4 (or Proposition 2) & $0 \leq \theta<1<\zeta$ & heads of $z$ & tails of $c z$ \\
\hline Corollary 5 (or Proposition 3) & $0 \leq \theta<1<\zeta$ & tails of $z$ & heads of $c z$ \\
\hline Corollary 6 (or Proposition 4) & $1<\theta \leq \zeta$ & tails of $z$ & tails of $c z$ \\
\hline
\end{tabular}

Similar to the discrete Hardy inequality (1.1), the continuous versions (1.2) or (1.3) have attracted many mathematicians' interests and expansions of these continuous inequalities have appeared in the literature. Continuous versions of discrete inequalities (1.4) (or (1.7)) and (1.5) (or (1.9)) were obtained by Copson [21, Theorem 1, Theorem 3], respectively. By these results, Copson derived better inequalities than Hardy's continuous inequalities (1.2) or (1.3) as follows.

Let $z$ and $h$ be nonnegative functions. We set $\bar{G}=\int_{0}^{t} z(s) d s, H(t)=\int_{0}^{t} z(s) h(s) d s$, $\bar{H}(t)=\int_{t}^{\infty} z(s) h(s) d s$.

If $1<\theta, 1 \leq \zeta, 0<b \leq \infty$, then

$$
\int_{0}^{b} \frac{z(t)[H(t)]^{\zeta}}{[\bar{G}(t)]^{\theta}} d t \leq\left(\frac{\zeta}{\theta-1}\right)^{\zeta} \int_{0}^{b} \frac{z(t) h^{\zeta}(t)}{[\bar{G}(t)]^{\theta-\zeta}} d t
$$

and if $\theta<1 \leq \zeta, a>0$, then

$$
\int_{a}^{\infty} \frac{z(t)[\bar{H}(t)]^{\zeta}}{[\bar{G}(t)]^{\theta}} d t \leq\left(\frac{\zeta}{1-\theta}\right)^{\zeta} \int_{a}^{\infty} \frac{z(t) h^{\zeta}(t)}{[\bar{G}(t)]^{\theta-\zeta}} d t
$$

Various generalizations and numerous variants of continuous Hardy-Copson inequalities (1.10) and (1.11) can be found in Pachpatte [54] and references therein. 
Other refinements of continuous Hardy-Copson inequalities, which are generalizations of (1.10) and (1.11), respectively, have been introduced by Pečarić and Hanjš [55] as in the following: Let $z$ and $h$ be nonnegative functions. We set $\bar{G}(t)=\int_{0}^{t} z(s) d s, H(t)=$ $\int_{0}^{t} z(s) h(s) d s, \bar{H}(t)=\int_{t}^{\infty} z(s) h(s) d s$. If $\zeta>1, \eta \geq 0, \eta+\theta>1$, then

$$
\int_{0}^{\infty} \frac{z(t)[H(t)]^{\eta+\zeta}}{[\bar{G}(t)]^{\eta+\theta}} d t \leq\left[\frac{\eta+\zeta}{\eta+\theta-1}\right]^{\zeta} \int_{0}^{\infty} \frac{z(t) h^{\zeta}(t)[H(t)]^{\eta}}{[\bar{G}(t)]^{\eta+\theta-\zeta}} d t
$$

and if $\zeta>1, \eta \geq 0, \eta+\theta<1$, then

$$
\int_{0}^{\infty} \frac{z(t)[\bar{H}(t)]^{\eta+\zeta}}{[\bar{G}(t)]^{\eta+\theta}} d t \leq\left[\frac{\eta+\zeta}{1-\eta-\theta}\right]^{\zeta} \int_{0}^{\infty} \frac{z(t) h^{\zeta}(t)[\bar{H}(t)]^{\eta}}{[\bar{G}(t)]^{\eta+\theta-\zeta}} d t .
$$

Following the development of the time scale concept $[8,15,16,25,26]$, the analysis of dynamic inequalities have become a popular research area and most classical inequalities have been extended to an arbitrary time scale. The surveys $[1,58]$ and the monograph [3] can be used to see these extended dynamic inequalities for delta approach. Although the nabla dynamic inequalities are less attractive compared to the delta ones, some of the nabla dynamic inequalities can be found in $[6,13,27,40,41,53,56]$.

The growing interest to Hardy-Copson type inequalities have taken place in the time scale calculus as well and delta unifications of these inequalities have been established in the book [4] and in the articles [59]-[65], [2,17, 18,22,23] whereas their nabla counterparts and extensions can be seen in [33-35].

The delta time scale generalizations of the foregoing Hardy-Copson type inequalities in an arbirtary time scale are given in the next four theorems.

A delta unification of the discrete Bennett's inequality (1.8) is stated as follows.

Theorem $1.1([64])$. Let $z$ and $h$ be nonnegative functions on $(0, \infty)_{\mathbb{T}^{\kappa}}$. We set $G_{1}(t)=$ $\int_{t}^{\infty} z(s) \Delta s$ and $H_{1}(t)=\int_{a}^{t} z(s) h(s) \Delta s$. If $\zeta>1, \eta \geq 0, \eta+\theta<1$, then we have

$$
\int_{a}^{\infty} \frac{z(t)\left[H_{1}^{\sigma}(t)\right]^{\eta+\zeta}}{\left[G_{1}(t)\right]^{\eta+\theta}} \Delta t \leq \frac{\eta+\zeta}{1-\eta-\theta} \int_{a}^{\infty} \frac{z(t) h(t)\left[H_{1}^{\sigma}(t)\right]^{\eta+\zeta-1}}{\left[G_{1}(t)\right]^{\eta+\theta-1}} \Delta t
$$

and

$$
\int_{a}^{\infty} \frac{z(t)\left[H_{1}^{\sigma}(t)\right]^{\eta+\zeta}}{\left[G_{1}(t)\right]^{\eta+\theta}} \Delta t \leq\left[\frac{\eta+\zeta}{1-\eta-\theta}\right]^{\zeta} \int_{a}^{\infty} \frac{z(t) h^{\zeta}(t)\left[H_{1}^{\sigma}(t)\right]^{\eta}}{\left[G_{1}(t)\right]^{\eta+\theta-\zeta}} \Delta t
$$

A delta unification of the Copson's discrete inequality (1.5) (or the Bennett's discrete inequality (1.9)) and its continuous versions (1.3), (1.11) as well as its continuous generalization (1.13) is stated as follows.

Theorem $1.2([64])$. Let $z$ and $h$ be nonnegative functions on $(0, \infty)_{\mathbb{T}^{\kappa}}$. We set $\bar{G}_{1}(t)=$ $\int_{a}^{t} z(s) \Delta s$ and $\bar{H}_{1}(t)=\int_{t}^{\infty} z(s) h(s) \Delta s$. If $\zeta>1, \eta \geq 0, \eta+\theta<1$, then we have

$$
\int_{a}^{\infty} \frac{z(t)\left[\bar{H}_{1}(t)\right]^{\eta+\zeta}}{\left[\bar{G}_{1}^{\sigma}(t)\right]^{\eta+\theta}} \Delta t \leq \frac{\eta+\zeta}{1-\eta-\theta} \int_{a}^{\infty} \frac{z(t) h(t)\left[\bar{H}_{1}(t)\right]^{\eta+\zeta-1}}{\left[\bar{G}_{1}^{\sigma}(t)\right]^{\eta+\theta-1}} \Delta t
$$

and

$$
\int_{a}^{\infty} \frac{z(t)\left[\bar{H}_{1}(t)\right]^{\eta+\zeta}}{\left[\bar{G}_{1}^{\sigma}(t)\right]^{\eta+\theta}} \Delta t \leq\left[\frac{\eta+\zeta}{1-\eta-\theta}\right]^{\zeta} \int_{a}^{\infty} \frac{z(t) h^{\zeta}(t)\left[\bar{H}_{1}(t)\right]^{\eta}}{\left[\bar{G}_{1}^{\sigma}(t)\right]^{\eta+\theta-\zeta}} \Delta t .
$$

A delta unification of the discrete Bennett's inequality (1.6) is established as in the folowing. 
Theorem $1.3([64])$. Let $z$ and $h$ be nonnegative functions on $(0, \infty)_{\mathbb{T}^{\kappa}}$. We set $G_{1}(t)=$ $\int_{t}^{\infty} z(s) \Delta s$ and $\bar{H}_{1}(t)=\int_{t}^{\infty} z(s) h(s) \Delta s$. For $\frac{G_{1}^{\sigma}(t)}{G_{1}(t)} \geq \frac{1}{K}>0$, if $\zeta>1, \eta \geq 0, \eta+\theta>1$, then we have

$$
\int_{a}^{\infty} \frac{z(t)\left[\bar{H}_{1}(t)\right]^{\eta+\zeta}}{\left[G_{1}(t)\right]^{\eta+\theta}} \Delta t \leq \frac{\eta+\zeta}{\eta+\theta-1} \int_{a}^{\infty} \frac{z(t) h(t)\left[\bar{H}_{1}(t)\right]^{\eta+\zeta-1}}{\left[G_{1}^{\sigma}(t)\right]^{\eta+\theta-1}} \Delta t
$$

and

$$
\int_{a}^{\infty} \frac{z(t)\left[\bar{H}_{1}(t)\right]^{\eta+\zeta}}{\left[G_{1}(t)\right]^{\eta+\theta}} \Delta t \leq\left[K^{\eta+\theta-1} \frac{\eta+\zeta}{\eta+\theta-1}\right]^{\zeta} \int_{a}^{\infty} \frac{z(t) h^{\zeta}(t)\left[\bar{H}_{1}(t)\right]^{\eta}}{\left[G_{1}(t)\right]^{\eta+\theta-\zeta}} \Delta t
$$

Although the authors presented Theorem 1.1-Theorem 1.3 in [64] for delta time scale calculus, they did not include the following theorem. For the completeness of the paper, we give the next theorem, which is a delta unification of the Hardy's discrete inequality (1.1), the Copson's discrete inequality (1.4) (or the Bennett's discrete inequality (1.7)) and of the continuous inequalities $(1.2),(1.3),(1.10)$ as well as of their continuous generalization (1.12), established in [33].

Theorem 1.4 ([33,64]). Let $z$ and $h$ be nonnegative functions on $(0, \infty)_{\mathbb{T}^{\kappa}}$. We set $\bar{G}_{1}(t)=$ $\int_{a}^{t} z(s) \Delta s$ and $H_{1}(t)=\int_{a}^{t} z(s) h(s) \Delta s$. For $\frac{\bar{G}_{1}(t)}{\bar{G}_{1}^{\sigma}(t)} \geq \frac{1}{J}>0$, if $\zeta>1, \eta \geq 0, \eta+\theta>1$, then we have

$$
\int_{a}^{\infty} \frac{z(t)\left[H_{1}^{\sigma}(t)\right]^{\eta+\zeta}}{\left[\bar{G}_{1}^{\sigma}(t)\right]^{\eta+\theta}} \Delta t \leq \frac{\eta+\zeta}{\eta+\theta-1} \int_{a}^{\infty} \frac{z(t) h(t)\left[H_{1}^{\sigma}(t)\right]^{\eta+\zeta-1}}{\left[\bar{G}_{1}(t)\right]^{\eta+\theta-1}} \Delta t
$$

and

$$
\int_{a}^{\infty} \frac{z(t)\left[H_{1}^{\sigma}(t)\right]^{\eta+\zeta}}{\left[\bar{G}_{1}^{\sigma}(t)\right]^{\eta+\theta}} \Delta t \leq\left[J^{\eta+\theta-1} \frac{\eta+\zeta}{\eta+\theta-1}\right]^{\zeta} \int_{a}^{\infty} \frac{z(t) h^{\zeta}(t)\left[H_{1}^{\sigma}(t)\right]^{\eta}}{\left[\bar{G}_{1}^{\sigma}(t)\right]^{\eta+\theta-\zeta}} \Delta t
$$

The construction of the nabla time scale calculus, which has been introduced simultaneously with the delta time scale calculus, can be found in $[8,15,16,25,26]$.

Contrary to delta case, nabla Hardy-Copson type inequalities have not been considered until 2021. The first results of this case were obtained by Kayar and Kaymakçalan in [33].

The nabla time scale generalizations of the foregoing inequalities in an arbirtary time scale are given in the next four theorems.

A nabla unification of the discrete Bennett's inequality (1.8) and a nabla analogue of the delta inequality (1.14) are stated as follows.

Theorem $1.5([33])$. Let $z$ and $h$ be nonnegative functions on $(0, \infty)_{\mathbb{T}_{\kappa}}$. We set $G_{2}(t)=$ $\int_{t}^{\infty} z(s) \nabla s$ and $H_{2}(t)=\int_{a}^{t} z(s) h(s) \nabla s$. Assume that $H_{2}(\infty)<\infty$ and $\int_{a}^{\infty} \frac{z(t) \nabla t}{\left[G_{2}^{\rho}(t)\right]^{\theta+\eta}}<$ $\infty$. If $\zeta>1, \eta \geq 0$ and $\eta+\theta<1$ are real constants, then we have

$$
\int_{a}^{\infty} \frac{z(t)\left[H_{2}(t)\right]^{\eta+\zeta}}{\left[G_{2}^{\rho}(t)\right]^{\eta+\theta}} \nabla t \leq \frac{\eta+\zeta}{1-\eta-\theta} \int_{a}^{\infty} \frac{z(t) h(t)\left[H_{2}(t)\right]^{\eta+\zeta-1}}{\left[G_{2}^{\rho}(t)\right]^{\eta+\theta-1}} \nabla t
$$

and

$$
\int_{a}^{\infty} \frac{z(t)\left[H_{2}(t)\right]^{\eta+\zeta}}{\left[G_{2}^{\rho}(t)\right]^{\eta+\theta}} \nabla t \leq\left[\frac{\eta+\zeta}{1-\eta-\theta}\right]^{\zeta} \int_{a}^{\infty} \frac{z(t) h^{\zeta}(t)\left[H_{2}(t)\right]^{\eta}}{\left[G_{2}^{\rho}(t)\right]^{\eta+\theta-\zeta}} \nabla t
$$

A nabla unification of the Copson's discrete inequality (1.5) (or the Bennett's discrete inequality (1.9)) and its continuous versions (1.3), (1.11) and (1.13) as well as a nabla analogue of the delta inequality (1.16) are stated as follows. 
Theorem 1.6 ([33]). Let $z$ and $h$ be nonnegative functions on $(0, \infty)_{\mathbb{T}_{\kappa}}$. We set $\bar{G}_{2}(t)=$ $\int_{a}^{t} z(s) \nabla s$ and $\bar{H}_{2}(t)=\int_{t}^{\infty} z(s) h(s) \nabla s$. Assume that $\bar{H}_{2}(a)<\infty$ and $\int_{a}^{\infty} \frac{z(t) \nabla t}{\left[\bar{G}_{2}^{\rho}(t)\right]^{\theta+\eta}}<$ $\infty$. If $\zeta>1, \eta \geq 0$ and $\eta+\theta<1$ are real constants, then we have

$$
\int_{a}^{\infty} \frac{z(t)\left[\bar{H}_{2}^{\rho}(t)\right]^{\eta+\zeta}}{\left[\bar{G}_{2}(t)\right]^{\eta+\theta}} \nabla t \leq \frac{\eta+\zeta}{1-\eta-\theta} \int_{a}^{\infty} \frac{z(t) h(t)\left[\bar{H}_{2}^{\rho}(t)\right]^{\eta+\zeta-1}}{\left[\bar{G}_{2}(t)\right]^{\eta+\theta-1}} \nabla t
$$

and

$$
\int_{a}^{\infty} \frac{z(t)\left[\bar{H}_{2}^{\rho}(t)\right]^{\eta+\zeta}}{\left[\bar{G}_{2}(t)\right]^{\eta+\theta}} \nabla t \leq\left[\frac{\eta+\zeta}{1-\eta-\theta}\right]^{\zeta} \int_{a}^{\infty} \frac{z(t) h^{\zeta}(t)\left[\bar{H}_{2}^{\rho}(t)\right]^{\eta}}{\left[\bar{G}_{2}(t)\right]^{\eta+\theta-\zeta}} \nabla t
$$

A nabla unification of the Bennett's discrete inequality (1.6) and a nabla analogue of the delta inequality (1.18) are stated as follows.

Theorem 1.7 ([33]). Let $z$ and $h$ be nonnegative functions on $(0, \infty)_{\mathbb{T}_{\kappa}}$. We set $G_{2}(t)=$ $\int_{t}^{\infty} z(s) \nabla s$ and $\bar{H}_{2}(t)=\int_{t}^{\infty} z(s) h(s) \nabla s$. Assume that $\bar{H}_{2}(a)<\infty$ and $\int_{a}^{\infty} \frac{z(t) \nabla t}{\left[G_{2}^{\rho}(t)\right]^{\theta+\eta}}<$ $\infty$. Suppose that there exists $M>0$ such that $\frac{G^{\rho}(t)}{G(t)} \leq M$ for $t \in(a, \infty)_{\mathbb{T}}$. If $\zeta>1, \eta \geq 0$ and $\eta+\theta>1$ are real constants, then we have

$$
\int_{a}^{\infty} \frac{z(t)\left[\bar{H}_{2}^{\rho}(t)\right]^{\eta+\zeta}}{\left[G_{2}^{\rho}(t)\right]^{\eta+\theta}} \nabla t \leq \frac{\eta+\zeta}{\eta+\theta-1} \int_{a}^{\infty} \frac{z(t) h(t)\left[\bar{H}_{2}^{\rho}(t)\right]^{\eta+\zeta-1}}{\left[G_{2}(t)\right]^{\eta+\theta-1}} \nabla t
$$

and

$$
\int_{a}^{\infty} \frac{z(t)\left[\bar{H}^{\rho}(t)\right]^{\eta+\zeta}}{\left[G^{\rho}(t)\right]^{\eta+\theta}} \nabla t \leq\left[M^{\eta+\theta-1} \frac{\eta+\zeta}{\eta+\theta-1}\right]^{\zeta} \int_{a}^{\infty} \frac{z(t) h^{\zeta}(t)\left[\bar{H}^{\rho}(t)\right]^{\eta}}{\left[G^{\rho}(t)\right]^{\eta+\theta-\zeta}} \nabla t .
$$

A nabla unification of the discrete inequalities (1.1), (1.4) and (1.7) and the continuous inequalities (1.2), (1.3), (1.10) and (1.12) as well as a nabla analogue of the delta inequality (1.20) are stated as follows.

Theorem 1.8 ([33]). Let $z$ and $h$ be nonnegative functions on $(0, \infty)_{\mathbb{T}_{\kappa}}$. We set $\bar{G}_{2}(t)=$ $\int_{a}^{t} z(s) \nabla s$ and $H_{2}(t)=\int_{a}^{t} z(s) h(s) \nabla s$. Assume that $H_{2}(\infty)<\infty$ and $\int_{a}^{\infty} \frac{z(t) \nabla t}{\left[\bar{G}_{2}(t)\right]^{\eta+\theta}}<$ $\infty$. Suppose that there exists $L>0$ such that $\frac{\bar{G}(t)}{\bar{G}^{\rho}(t)} \leq L$ for $t \in(a, \infty)_{\mathbb{T}}$. If $\zeta>1, \eta \geq 0$ and $\eta+\theta>1$ are real constants, then we have

$$
\int_{a}^{\infty} \frac{z(t)\left[H_{2}(t)\right]^{\eta+\zeta}}{\left[\bar{G}_{2}(t)\right]^{\eta+\theta}} \nabla t \leq \frac{\eta+\zeta}{\eta+\theta-1} \int_{a}^{\infty} \frac{z(t) h(t)\left[H_{2}(t)\right]^{\eta+\zeta-1}}{\left[\bar{G}_{2}^{\rho}(t)\right]^{\eta+\theta-1}} \nabla t
$$

and

$$
\int_{a}^{\infty} \frac{z(t)[H(t)]^{\eta+\zeta}}{[\bar{G}(t)]^{\eta+\theta}} \nabla t \leq\left[L^{\eta+\theta-1} \frac{\eta+\zeta}{\eta+\theta-1}\right]^{\zeta} \int_{a}^{\infty} \frac{z(t) h^{\zeta}(t)[H(t)]^{\eta}}{[\bar{G}(t)]^{\eta+\theta-\zeta}} \nabla t .
$$

To the best of our knowledge, contrary to delta and nabla cases, diamond alpha HardyCopson type inequalities have not been considered yet. Hence the main contributions of this article are to bind and unify abovementioned Hardy-Copson type inequalities by diamond alpha calculus and to obtain new inequalities even for the discrete, continuous, delta and nabla cases by taking account of the condition $\eta+\theta \leq 0$, which has not been considered so far. We notice that there is more than one way to obtain diamond alpha Hardy-Copson type inequalities. Our first method is inspired from the papers [64] and [33] and is based on the convex linear combination of the aforementioned delta and nabla Hardy-Copson type inequalities given in [33,64]. By this method, we establish HardyCopson type integral inequalities whose left or right hand side consists of delta and nabla 
integrals and right or left hand side composed of diamond alpha integrals, respectively. Our second approach, which is a novel method for the diamond alpha calculus, is to obtain diamond alpha versions of Theorem 1.1-Theorem 1.8 by using diamond alpha calculus rather than algebra. By this method, since both sides of the diamond alpha HardyCopson type inequalities include only single diamond alpha integrals, these inequalities become compact forms. In this case, we have twelve diamond alpha Hardy-Copson type inequalities, some of which are new even for delta and nabla time scale calculi while the others are fusions of the results obtained for such calculi and all of which merge the aforementioned inequalities. As a result, these techniques allow us to generalize and unify the foregoing delta and nabla Hardy-Copson type inequalities and to obtain novel inequalities for delta and nabla approaches as well as to contribute the current literature by new diamond alpha Hardy-Copson type inequalities.

The organization of this paper can be seen as follows. Delta, nabla and diamond alpha time scale calculi and their main propoerties are introduced in Section 2. The contribution of Section 3, which includes one of the main results, is to unify the recently developed results presented in $[33,64]$ for time scale diamond alpha calculus by combining dynamic delta and nabla integral inequalities, both of which are special cases of dynamic diamond alpha integral inequalities. Another main result of this paper is given in Section 4 which contains diamond alpha unifications of the foregoing dynamic delta and nabla integral inequalities proven in Theorem 1.1-Theorem 1.8.

\section{Preliminaries}

This section is devoted to present the main definitions and theorems of delta, nabla and diamond alpha calculi. We refer the reader to $[8,15,16]$ for the concept of time scale calculus in delta and nabla senses.

A nonempty closed subset of $\mathbb{R}$ is called a time scale which is denoted by $\mathbb{T}$. Since the delta and the nabla time scale calculi are very well-known $[8,15,16]$, we skip the details of them and we consider only the main properties which will be used in the sequel.

Theorem 2.1 ([15]). Suppose that $\Lambda, \Gamma: \mathbb{T} \rightarrow \mathbb{R}$ and $s \in \mathbb{T}^{\kappa}$. For $\mu(s)=\sigma(s)-s$, we have the following.

(1) If $\Lambda$ is delta differentiable at $s$, then $\Lambda(\sigma(s))=\Lambda^{\sigma}(s)=\Lambda(s)+\mu(s) \Lambda^{\Delta}(s)$.

(2) The product $\Lambda \Gamma: \mathbb{T} \rightarrow \mathbb{R}$ is differentiable at $s$ with

$$
(\Lambda \Gamma)^{\Delta}(s)=\Lambda^{\Delta}(s) \Gamma(s)+\Lambda(\sigma(s)) \Gamma^{\Delta}(s)=\Lambda(s) \Gamma^{\Delta}(s)+\Lambda^{\Delta}(s) \Gamma(\sigma(s)) .
$$

Lemma $2.2([15])$. [Chain rules for delta derivative] If $\Gamma \in C^{1}(\mathbb{R}, \mathbb{R})$ and $\Lambda \in C(\mathbb{T}, \mathbb{R})$ is delta differentiable on $\mathbb{T}^{\kappa}$, then $\Gamma \circ \Lambda$ is delta differentiable and

(i) one can find $c \in[s, \sigma(s)]$ with

$$
(\Gamma \circ \Lambda)^{\Delta}(s)=\Gamma^{\prime}(\Lambda(c)) \Lambda^{\Delta}(s) .
$$

(ii) the equation

$$
(\Gamma \circ \Lambda)^{\Delta}(s)=\Lambda^{\Delta}(s) \int_{0}^{1} \Gamma^{\prime}\left(\Lambda(s)+w \mu(s) \Lambda^{\Delta}(s)\right) d w
$$

holds.

Theorem 2.3 ([15]). Suppose that $\Gamma: \mathbb{T} \rightarrow \mathbb{R}$ and $s \in \mathbb{T}_{\kappa}$. For $\nu(s)=s-\rho(s)$, we have the following.

(1) If $\Gamma$ is nabla differentiable at $s$, then $\Gamma(\rho(s))=\Gamma^{\rho}(s)=\Gamma(s)-\nu(s) \Gamma^{\nabla}(s)$.

(2) The product $\Lambda \Gamma: \mathbb{T} \rightarrow \mathbb{R}$ is differentiable at $s$ with

$$
(\Lambda \Gamma)^{\nabla}(s)=\Lambda^{\nabla}(s) \Gamma(s)+\Lambda(\rho(s)) \Gamma^{\nabla}(s)=\Lambda(s) \Gamma^{\nabla}(s)+\Lambda^{\nabla}(s) \Gamma(\rho(s)) .
$$


Lemma 2.4 ([27]). [Chain rule for nabla derivative] If $\Gamma \in C^{1}(\mathbb{R}, \mathbb{R})$ and $\Lambda \in C(\mathbb{T}, \mathbb{R})$ is nabla differentiable on $\mathbb{T}_{\kappa}$, then $\Gamma \circ \Lambda$ is nabla differentiable and

$$
(\Gamma \circ \Lambda)^{\nabla}(s)=\Lambda^{\nabla}(s) \int_{0}^{1} \Gamma^{\prime}\left(\Lambda(\rho(s))+w \nu(s) \Lambda^{\nabla}(s)\right) d w
$$

holds.

The next lemmas play crucial roles in the main theorems.

Lemma 2.5 ([8,15]). If $\Gamma$ is continuous, then
(i) $\left(\int_{t_{1}}^{t} \Gamma(s) \Delta s\right)^{\Delta}=\Gamma(t)$ and $\left(\int_{t_{1}}^{t} \Gamma(s) \nabla s\right)^{\Delta}=\Gamma(\sigma(t))$
(ii) $\left(\int_{t_{1}}^{t} \Gamma(s) \nabla s\right)^{\nabla}=\Gamma(t)$ and $\left(\int_{t_{1}}^{t} \Gamma(s) \Delta s\right)^{\nabla}=\Gamma(\rho(t))$

Lemma 2.6 ([26]). If $\Gamma$ is continuous for all $t_{1}, t_{2} \in \mathbb{T}$ with $t_{1}<t_{2}$, then

$$
\int_{t_{1}}^{t_{2}} \Gamma(t) \Delta t=\int_{t_{1}}^{t_{2}} \Gamma(\rho(t)) \nabla t \quad \text { and } \quad \int_{t_{1}}^{t_{2}} \Gamma(t) \nabla t=\int_{t_{1}}^{t_{2}} \Gamma(\sigma(t)) \Delta t .
$$

The diamond alpha time scale calculus has been introduced by Sheng et al. in the article [66]. This calculus deals with diamond alpha, which is denoted by $\diamond_{\alpha}$, differentiable and diamond alpha integrable functions which are convex linear combinations of delta and nabla differentiable and integrable functions, respectively. For some developments of this calculus and for some integral inequalities in this calculus, we refer to $[5,7,12-14,32,36-$ $39,48,50,51,53,57]$ and references therein.

Let $\rho(s)-\tau=a_{s \tau}$ and $\sigma(s)-\tau=b_{s \tau}$. Then the $\diamond_{\alpha}$-derivative of $\Lambda: \mathbb{T} \rightarrow \mathbb{R}$ at the point $s \in \mathbb{T}_{\kappa}^{\kappa}$ denoted by $\Lambda^{\diamond}(s)$ is the number enjoying the property that for all $\epsilon>0$, there exists a neighborhood $V \subset \mathbb{T}$ of $s \in \mathbb{T}_{\kappa}^{\kappa}$ such that for any $\tau \in V$,

$|\alpha| \Lambda(\sigma(s))-\Lambda(\tau)|| a_{s \tau}|+(1-\alpha)| \Lambda(\rho(s))-\Lambda(\tau)|| b_{s \tau}\left|-\Lambda^{\diamond_{\alpha}}(s)\right| a_{s \tau}|| b_{s \tau}|| \leq \epsilon\left|a_{s \tau}\right|\left|b_{s \tau}\right|$.

By [66], $\Lambda: \mathbb{T} \rightarrow \mathbb{R}$ is $\diamond_{\alpha}$-differentiable at $s \in \mathbb{T}_{\kappa}^{\kappa}$ provided it is both delta and nabla differentiable at $s$. Moreover, for $0 \leq \alpha \leq 1$, such a function satisfies

$$
\Lambda^{\diamond_{\alpha}}(s)=\alpha \Lambda^{\Delta}(s)+(1-\alpha) \Lambda^{\nabla}(s) .
$$

By [66], $\Lambda: \mathbb{T} \rightarrow \mathbb{R}$ is diamond alpha integrable provided it is continuous. Moreover, for $0 \leq \alpha \leq 1$, we have

$$
\int_{s_{1}}^{s_{2}} \Lambda(s) \diamond_{\alpha} s=\alpha \int_{s_{1}}^{s_{2}} \Lambda(s) \Delta s+(1-\alpha) \int_{s_{1}}^{s_{2}} \Lambda(s) \nabla s .
$$

Lemma $2.7([26,51])$. For all $s \in \mathbb{T}$, a time scale $\mathbb{T}$ is said to be regular provided $\sigma(\rho(s))=$ $\rho(\sigma(s))=s$ holds. A regular time scale $\mathbb{T}$ satisfies $\mathbb{T}_{\kappa}^{\kappa}=\mathbb{T}_{\kappa}=\mathbb{T}^{\kappa}=\mathbb{T}$. Moreover, $\sigma(\mathbb{T})=\rho(\mathbb{T})=\mathbb{T}$ in such a time scale.

Lemma 2.8 (Diamond-alpha Hölder's inequality). [5,53] Let $s_{1}, s_{2} \in \mathbb{T}$. For $\Lambda, \Gamma \in$ $C\left([a, b]_{\mathbb{T}},[0, \infty)\right)$ with $\int_{s_{1}}^{s_{2}} \Gamma^{\lambda_{2}}(s) \diamond_{\alpha} s>0$, and for the conjugate numbers $\lambda_{1}, \lambda_{2}>1$ satisfying $\frac{1}{\lambda_{1}}+\frac{1}{\lambda_{2}}=1$, the following Hölder's inequality

$$
\int_{s_{1}}^{s_{2}} \Lambda(s) \Gamma(s) \diamond_{\alpha} s \leq\left(\int_{s_{1}}^{s_{2}} \Lambda^{\lambda_{1}}(s) \diamond_{\alpha} s\right)^{1 / \lambda_{1}}\left(\int_{s_{1}}^{s_{2}} \Gamma^{\lambda_{2}}(s) \diamond_{\alpha} s\right)^{1 / \lambda_{2}}
$$

holds. 
One of the disadvantages of diamond alpha integral which sometimes does not allow us to use diamond alpha calculus is that the fundamental theorem of calculus is not fulfilled for diamond alpha integral. However, by [66], it is known that

$$
\left(\int_{t_{1}}^{t} \Lambda(s) \diamond_{\alpha} s\right)^{\diamond_{\alpha}}=\left(1-2 \alpha-\alpha^{2}\right) \Lambda(t)+\left(\alpha-\alpha^{2}\right)[\Lambda(\rho(t))+\Lambda(\sigma(t))] .
$$

\section{Dynamic diamond alpha inequalities-I}

Let $\mathbb{T}$ be a time scale and $a \in[0, \infty)_{\mathbb{T}}$. This section is devoted to find new integral inequalities, which are established by using the second inequalities (the first inequalities can be used as well) in Theorem 1.1-Theorem 1.8. In addition to their novelty, these inequalities cover the ones in Theorem 1.1-Theorem 1.8. Since the following inequalities follow from the fact that diamond alpha integral is the convex linear combination of delta and nabla integrals, these inequalities can be considered as mixed type Hardy-Copson diamond alpha inequalities.

The next theorem provides diamond alpha unifications of the previous Hardy-Copson type inequalities given for $\zeta>1, \eta \geq 0$ and $0 \leq \eta+\theta<1$. These previous Hardy-Copson type inequalities are listed as follows:

(a) The discrete inequality (1.8) obtained by Bennett [11, Corollary 5] or Leindler [45, Proposition 3].

(b) The continuous inequality obtained by Saker et al. [64, Corollary 2.2] or Kayar and Kaymakçalan [33, Remark 3.22].

(c) The delta inequality (1.15) obtained by Saker et al. [64, Theorem 2.2].

(d) The nabla inequality (1.23) obtained by Kayar and Kaymakçalan [33, Theorem 3.19].

Theorem 3.1. Suppose that $z$ and $h$ are nonnegative functions on $\mathbb{T}$. Let $G_{1}(t)=$ $\int_{t}^{\infty} z(s) \Delta s, G_{2}(t)=\int_{t}^{\infty} z(s) \nabla s, H_{1}(t)=\int_{a}^{t} z(s) h(s) \Delta s$ and $H_{2}(t)=\int_{a}^{t} z(s) h(s) \nabla s$. Moreover let us define $H(t)=\min _{t \in[a, \infty)}\left\{H_{1}^{\sigma}(t), H_{2}(t)\right\}, G(t)=\max _{t \in[a, \infty)}\left\{G_{1}(t), G_{2}^{\rho}(t)\right\}$, and $\widetilde{H}(t)=\max _{t \in[a, \infty)}\left\{H_{1}^{\sigma}(t), H_{2}(t)\right\}$. If $\zeta>1, \eta \geq 0$ and $0 \leq \eta+\theta<1$ are real constants, then

(i)

$$
\begin{aligned}
\int_{a}^{\infty} \frac{z(t)[H(t)]^{\eta+\zeta}}{[G(t)]^{\eta+\theta}} \diamond_{\alpha} t & \leq\left[\frac{\eta+\zeta}{1-\eta-\theta}\right]^{\zeta} \\
& \times\left[\int_{a}^{\infty} \frac{z(t) h^{\zeta}(t)\left[H_{1}^{\sigma}(t)\right]^{\eta}}{\left[G_{1}(t)\right]^{\eta+\theta-\zeta}} \Delta t+\int_{a}^{\infty} \frac{z(t) h^{\zeta}(t)\left[H_{2}(t)\right]^{\eta}}{\left[G_{2}^{\rho}(t)\right]^{\eta+\theta-\zeta}} \nabla t\right] .
\end{aligned}
$$

(ii)

$$
\begin{aligned}
\alpha \int_{a}^{\infty} \frac{z(t)\left[H_{1}^{\sigma}(t)\right]^{\eta+\zeta}}{\left[G_{1}(t)\right]^{\eta+\theta}} \Delta t & +(1-\alpha) \int_{a}^{\infty} \frac{z(t)\left[H_{2}(t)\right]^{\eta+\zeta}}{\left[G_{2}^{\rho}(t)\right]^{\eta+\theta}} \nabla t \\
& \leq\left[\frac{\eta+\zeta}{1-\eta-\theta}\right]^{\zeta} \int_{a}^{\infty} \frac{z(t) h^{\zeta}(t)[\widetilde{H}(t)]^{\eta}}{[G(t)]^{\eta+\theta-\zeta}} \diamond_{\alpha} t
\end{aligned}
$$

(iii)

$$
\int_{a}^{\infty} \frac{z(t)[H(t)]^{\eta+\zeta}}{[G(t)]^{\eta+\theta}} \diamond_{\alpha} t \leq\left[\frac{\eta+\zeta}{1-\eta-\theta}\right]^{\zeta} \int_{a}^{\infty} \frac{z(t) h^{\zeta}(t)[\widetilde{H}(t)]^{\eta}}{[G(t)]^{\eta+\theta-\zeta}} \diamond_{\alpha} t
$$


Proof. (i) It follows from inequality (1.15) for $0 \leq \alpha \leq 1$ that

$$
\begin{aligned}
\alpha \int_{a}^{\infty} \frac{z(t)[H(t)]^{\eta+\zeta}}{[G(t)]^{\eta+\theta}} \Delta t & \leq \alpha \int_{a}^{\infty} \frac{z(t)\left[H_{1}^{\sigma}(t)\right]^{\eta+\zeta}}{\left[G_{1}(t)\right]^{\eta+\theta}} \Delta t \leq \int_{a}^{\infty} \frac{z(t)\left[H_{1}^{\sigma}(t)\right]^{\eta+\zeta}}{\left[G_{1}(t)\right]^{\eta+\theta}} \Delta t \\
& \leq\left[\frac{\eta+\zeta}{1-\eta-\theta}\right]^{\zeta} \int_{a}^{\infty} \frac{z(t) h^{\zeta}(t)\left[H_{1}^{\sigma}(t)\right]^{\eta}}{\left[G_{1}(t)\right]^{\eta+\theta-\zeta}} \Delta t
\end{aligned}
$$

Similarly, inequality (1.23) implies for $0 \leq \alpha \leq 1$ that

$$
\begin{aligned}
(1-\alpha) \int_{a}^{\infty} \frac{z(t)[H(t)]^{\eta+\zeta}}{[G(t)]^{\eta+\theta}} \nabla t & \leq(1-\alpha) \int_{a}^{\infty} \frac{z(t)\left[H_{2}(t)\right]^{\eta+\zeta}}{\left[G_{2}^{\rho}(t)\right]^{\eta+\theta}} \nabla t \leq \int_{a}^{\infty} \frac{z(t)\left[H_{2}(t)\right]^{\eta+\zeta}}{\left[G_{2}^{\rho}(t)\right]^{\eta+\theta}} \nabla t \\
& \leq\left[\frac{\eta+\zeta}{1-\eta-\theta}\right]^{\zeta} \int_{a}^{\infty} \frac{z(t) h^{\zeta}(t)\left[H_{2}(t)\right]^{\eta}}{\left[G_{2}^{\rho}(t)\right]^{\eta+\theta-\zeta}} \nabla t .
\end{aligned}
$$

If we add inequalities (3.3) and (3.4) side by side, we obtain the desired result.

(ii) Multiplying both sides of inequality (1.15) and inequality (1.23) by $\alpha$ and $1-\alpha$, respectively, and adding the resulting inequalities side by side lead to

$$
\begin{aligned}
\alpha \int_{a}^{\infty} \frac{z(t)\left[H_{1}^{\sigma}(t)\right]^{\eta+\zeta} \Delta t}{\left[G_{1}(t)\right]^{\eta+\theta}} & +(1-\alpha) \int_{a}^{\infty} \frac{z(t)\left[H_{2}(t)\right]^{\eta+\zeta} \nabla t}{\left[G_{2}^{\rho}(t)\right]^{\eta+\theta}} \\
& \leq \alpha\left[\frac{\eta+\zeta}{1-\eta-\theta}\right]^{\zeta} \int_{a}^{\infty} \frac{z(t) h^{\zeta}(t)\left[H_{1}^{\sigma}(t)\right]^{\eta}}{\left[G_{1}(t)\right]^{\eta+\theta-\zeta}} \Delta t \\
& +(1-\alpha)\left[\frac{\eta+\zeta}{1-\eta-\theta}\right]^{\zeta} \int_{a}^{\infty} \frac{z(t) h^{\zeta}(t)\left[H_{2}(t)\right]^{\eta}}{\left[G_{2}^{\rho}(t)\right]^{\eta+\theta-\zeta}} \nabla t
\end{aligned}
$$

By using $G(t)=\max _{t \in[a, \infty)}\left\{G_{1}(t), G_{2}^{\rho}(t)\right\}$ and $\widetilde{H}(t)=\max _{t \in[a, \infty)}\left\{H_{1}^{\sigma}(t), H_{2}(t)\right\}$ for $\eta+\theta-\zeta \leq 0$, on the right hand side of inequality (3.5), we get the desired result.

(iii) If we use $H(t)=\min _{t \in[a, \infty)}\left\{H_{1}^{\sigma}(t), H_{2}(t)\right\}$ and $G(t)=\max _{t \in[a, \infty)}\left\{G_{1}(t), G_{2}^{\rho}(t)\right\}$ on the left hand side of the inequality (3.1), we arrive the desired result.

Remark 3.2. If we choose $\alpha=1$ and $\alpha=0$ in inequality (3.5), then we obtain

$$
\int_{a}^{\infty} \frac{z(t)\left[H_{1}^{\sigma}(t)\right]^{\eta+\zeta}}{\left[G_{1}(t)\right]^{\eta+\theta}} \Delta t \leq\left[\frac{\eta+\zeta}{1-\eta-\theta}\right]^{\zeta} \int_{a}^{\infty} \frac{z(t) h^{\zeta}(t)\left[H_{1}^{\sigma}(t)\right]^{\eta}}{\left[G_{1}(t)\right]^{\eta+\theta-\zeta}} \Delta t
$$

and

$$
\int_{a}^{\infty} \frac{z(t)\left[H_{2}(t)\right]^{\eta+\zeta}}{\left[G_{2}^{\rho}(t)\right]^{\eta+\theta}} \nabla t \leq\left[\frac{\eta+\zeta}{1-\eta-\theta}\right]^{\zeta} \int_{a}^{\infty} \frac{z(t) h^{\zeta}(t)\left[H_{2}(t)\right]^{\eta}}{\left[G_{2}^{\rho}(t)\right]^{\eta+\theta-\zeta}} \nabla t
$$

which are exactly the same as the results of Theorem 1.1 and Theorem 1.5, respectively. Therefore the result in (ii) is the diamond alpha unification of the delta Hardy-Copson inequality (1.15) and the nabla Hardy-Copson inequality (1.23).

Remark 3.3. Although inequality (3.1) is better than inequality (3.2), it is worth mentioning inequality (3.2) due to the fact that the mixed integrals disappear and there exist only diamond alpha integrals on the both sides of the inequality (3.2).

The next theorem provides diamond alpha unifications of the previous Hardy-Copson type inequalities given for $\zeta>1, \eta \geq 0$ and $0 \leq \eta+\theta<1$. These previous Hardy-Copson type inequalities are listed as follows:

(a) The discrete inequality (1.5) obtained by Copson [20, Theorem 2.1] and the discrete inequality (1.9) obtained by Bennett [11, Corollary 4] or Leindler [45, Proposition $3]$. 
(b) The continuous inequality (1.3) obtained by Hardy et al. [30, Theorem 330], the continuous inequality (1.11) obtained by Copson [21, Theorem 3] and the continuous inequality (1.13) obtained by Pachpatte as inequality (6) in [54, Theorem 1] and Pečarić and Hanjš as inequality (9) in [55, Theorem 3].

(c) The delta inequality (1.17) obtained by Saker et al. [64, Theorem 2.1].

(d) The nabla inequality (1.25) obtained by Kayar and Kaymakçalan [33, Theorem 3.13].

Theorem 3.4. Suppose that $z$ and $h$ are nonnegative functions. Let $\overline{G_{1}}(t)=\int_{a}^{t} z(s) \Delta s$, $\overline{G_{2}}(t)=\int_{a}^{t} z(s) \nabla s, \overline{H_{1}}(t)=\int_{t}^{\infty} z(s) f(s) \Delta s$ and $\overline{H_{2}}(t)=\int_{t}^{\infty} z(s) h(s) \nabla s$. Moreover let us define $\bar{H}(t)=\min _{t \in[a, \infty)}\left\{\bar{H}_{1}(t), \bar{H}_{2}^{\rho}(t)\right\}, \bar{G}(t)=\max _{t \in[a, \infty)}\left\{\bar{G}_{1}^{\sigma}(t), \bar{G}_{2}(t)\right\}$ and $\tilde{\bar{H}}(t)=$ $\max _{t \in[a, \infty)}\left\{\bar{H}_{1}(t), \bar{H}_{2}^{\rho}(t)\right\}$. If $\zeta>1, \eta \geq 0$ and $0 \leq \eta+\theta<1$ are real constants, then

(i)

$$
\begin{aligned}
\int_{a}^{\infty} \frac{z(t)[\bar{H}(t)]^{\eta+\zeta}}{[\bar{G}(t)]^{\eta+\theta}} \diamond_{\alpha} t & \leq\left[\frac{\eta+\zeta}{1-\eta-\theta}\right]^{\zeta} \\
& \times\left[\int_{a}^{\infty} \frac{z(t) h^{\zeta}(t)\left[\bar{H}_{1}(t)\right]^{\eta}}{\left[\bar{G}_{1}^{\sigma}(t)\right]^{\eta+\theta-\zeta}} \Delta t+\int_{a}^{\infty} \frac{z(t) h^{\zeta}(t)\left[\bar{H}_{2}^{\rho}(t)\right]^{\eta}}{\left[\bar{G}_{2}(t)\right]^{\eta+\theta-\zeta}} \nabla t\right] .
\end{aligned}
$$

(ii)

(iii)

$$
\begin{aligned}
\alpha \int_{a}^{\infty} \frac{z(t)\left[\bar{H}_{1}(t)\right]^{\eta+\zeta}}{\left[\bar{G}_{1}^{\sigma}(t)\right]^{\eta+\theta}} \Delta t & +(1-\alpha) \int_{a}^{\infty} \frac{z(t)\left[\bar{H}_{2}^{\rho}(t)\right]^{\eta+\zeta}}{\left[\bar{G}_{2}(t)\right]^{\eta+\theta}} \nabla t \\
& \leq\left[\frac{\eta+\zeta}{1-\eta-\theta}\right]^{\zeta} \int_{a}^{\infty} \frac{z(t) h^{\zeta}(t)[\tilde{\bar{H}}(t)]^{\eta}}{[\bar{G}(t)]^{\eta+\theta-\zeta}} \diamond_{\alpha} t
\end{aligned}
$$

$$
\int_{a}^{\infty} \frac{z(t)[\bar{H}(t)]^{\eta+\zeta}}{[\bar{G}(t)]^{\eta+\theta}} \diamond_{\alpha} t \leq\left[\frac{\eta+\zeta}{1-\eta-\theta}\right]^{\zeta} \int_{a}^{\infty} \frac{z(t) h^{\zeta}(t)[\tilde{\bar{H}}(t)]^{\eta}}{[\bar{G}(t)]^{\eta+\theta-\zeta}} \diamond_{\alpha} t .
$$

Proof. If the same steps of the proof of Theorem 3.1 are followed for the delta ineqality (1.17) and the nabla inequality (1.25), the proof will be completed.

Remark 3.5. After expressing the right hand side of the inequality (3.6) in terms of delta and nabla integrals, if we choose $\alpha=1$ and $\alpha=0$ in the resulting inequality, then we obtain

and

$$
\int_{a}^{\infty} \frac{z(t)\left[\bar{H}_{1}(t)\right]^{\eta+\zeta}}{\left[\bar{G}_{1}^{\sigma}(t)\right]^{\eta+\theta}} \Delta t \leq\left[\frac{\eta+\zeta}{1-\eta-\theta}\right]^{\zeta} \int_{a}^{\infty} \frac{z(t) h^{\zeta}(t)\left[\bar{H}_{1}(t)\right]^{\eta}}{\left[\bar{G}_{1}^{\sigma}(t)\right]^{\eta+\theta-\zeta}} \Delta t
$$

$$
\int_{a}^{\infty} \frac{z(t)\left[\bar{H}_{2}^{\rho}(t)\right]^{\eta+\zeta}}{\left[\bar{G}_{2}(t)\right]^{\eta+\theta}} \nabla t \leq\left[\frac{\eta+\zeta}{1-\eta-\theta}\right]^{\zeta} \int_{a}^{\infty} \frac{z(t) h^{\zeta}(t)\left[\bar{H}_{2}^{\rho}(t)\right]^{\eta}}{\left[\bar{G}_{2}(t)\right]^{\eta+\theta-\zeta}} \nabla t
$$

which are exactly the same as the results of Theorem 1.2 and Theorem 1.6, respectively. Therefore the result in (ii) is the diamond alpha unification of the delta Hardy-Copson inequality (1.17) and the nabla Hardy-Copson inequality (1.25).

Remark 3.6. Although inequality (3.6) is better than inequality (3.7), it is worth mentioning inequality (3.7) due to the fact that the mixed integrals disappear and there exist only diamond alpha integrals on the both sides of the inequality (3.7).

The next theorem provides diamond alpha unifications of the previous Hardy-Copson type inequalities given for $\zeta>1, \eta \geq 0$ and $\eta+\theta>1$. These previous Hardy-Copson type inequalities are listed as follows: 
(a) The discrete inequality (1.6) obtained by Bennett [11, Corollary 6] or Leindler [45, Proposition 4].

(b) The continuous inequality obtained by Saker et al. [64, Corollary 2.3] and by Kayar and Kaymakçalan [33, Remark 3.11].

(c) The delta inequality (1.19) obtained by Saker et al. [64, Theorem 2.3].

(d) The nabla inequality (1.27) obtained by Kayar and Kaymakçalan [33, Theorem 3.8].

Theorem 3.7. Let the fuctions $G_{1}, G_{2}, \overline{H_{1}}, \overline{H_{2}}, G, \bar{H}, \widetilde{H}$ and be defined as in Theorem 3.1 and Theorem 3.4. Let $\frac{G_{1}^{\sigma}(t)}{G_{1}(t)} \geq \frac{1}{K_{1}}>0$ and $\frac{G_{2}(t)}{G_{2}^{\rho}(t)} \geq \frac{1}{K_{2}}>0$ and $K=\max \left\{K_{1}, K_{2}\right\}$. If $\zeta>1, \eta \geq 0$ and $\eta+\theta>1$ are real constants, then

(i)

$$
\begin{aligned}
\int_{a}^{\infty} \frac{z(t)[\bar{H}(t)]^{\eta+\zeta}}{[G(t)]^{\eta+\theta}} \diamond_{\alpha} t & \leq\left[K_{1}^{\eta+\theta-1} \frac{\eta+\zeta}{\eta+\theta-1}\right]^{\zeta} \int_{a}^{\infty} \frac{z(t) h^{\zeta}(t)\left[\bar{H}_{1}(t)\right]^{\eta}}{\left[G_{1}(t)\right]^{\eta+\theta-\zeta}} \Delta t \\
& +\left[K_{2}^{\eta+\theta-1} \frac{\eta+\zeta}{\eta+\theta-1}\right]^{\zeta} \int_{a}^{\infty} \frac{z(t) h^{\zeta}(t)\left[\bar{H}_{2}^{\rho}(t)\right]^{\eta}}{\left[G_{2}^{\rho}(t)\right]^{\eta+\theta-\zeta}} \nabla t
\end{aligned}
$$

(ii)

$$
\begin{aligned}
\alpha \int_{a}^{\infty} \frac{z(t)\left[\bar{H}_{1}(t)\right]^{\eta+\zeta}}{\left[G_{1}(t)\right]^{\eta+\theta}} \Delta t & +(1-\alpha) \int_{a}^{\infty} \frac{z(t)\left[\bar{H}_{2}^{\rho}(t)\right]^{\eta+\zeta}}{\left[G_{2}^{\rho}(t)\right]^{\eta+\theta}} \nabla t \\
& \leq\left[K^{\eta+\theta-1} \frac{\eta+\zeta}{\eta+\theta-1}\right]^{\zeta} \int_{a}^{\infty} \frac{z(t) h^{\zeta}(t)[\tilde{\bar{H}}(t)]^{\eta}}{[G(t)]^{\eta+\theta-\zeta}} \diamond_{\alpha} t
\end{aligned}
$$

(iii)

$$
\int_{a}^{\infty} \frac{z(t)[\bar{H}(t)]^{\eta+\zeta}}{[G(t)]^{\eta+\theta}} \diamond_{\alpha} t \leq\left[K^{\eta+\theta-1} \frac{\eta+\zeta}{\eta+\theta-1}\right]^{\zeta} \int_{a}^{\infty} \frac{z(t) h^{\zeta}(t)[\tilde{\bar{H}}(t)]^{\eta}}{[G(t)]^{\eta+\theta-\zeta}} \diamond_{\alpha} t
$$

Proof. If we follow the same procedure of the proof of Theorem 3.1 for the delta inequality (1.19) and the nabla inequality (1.27), we obtain the desired result.

Remark 3.8. If $1<\zeta \leq \eta+\theta$, then $G(t)$ can be replaced by $\widetilde{G}(t)=\min _{t \in[a, \infty)}\left\{G_{1}(t), G_{2}^{\rho}(t)\right\}$ on the right hand sides of the inequalities (3.8) and (3.9).

Remark 3.9. After expressing the right hand side of the inequality (3.8) in terms of delta and nabla integrals, if we choose $\alpha=1$ and $\alpha=0$ in the resulting inequality, then we obtain

$$
\int_{a}^{\infty} \frac{z(t)\left[\bar{H}_{1}(t)\right]^{\eta+\zeta}}{\left[G_{1}^{\sigma}(t)\right]^{\eta+\theta}} \Delta t \leq\left[K_{1}^{\eta+\theta-1} \frac{\eta+\zeta}{\eta+\theta-1}\right]^{\zeta} \int_{a}^{\infty} \frac{z(t) h^{\zeta}(t)\left[\bar{H}_{1}(t)\right]^{\eta}}{\left[\bar{G}_{1}(t)\right]^{\eta+\theta-\zeta}} \Delta t
$$

and

$$
\int_{a}^{\infty} \frac{z(t)\left[\bar{H}_{2}^{\rho}(t)\right]^{\eta+\zeta}}{\left[G_{2}^{\rho}(t)\right]^{\eta+\theta}} \nabla t \leq\left[K_{2}^{\eta+\theta-1} \frac{\eta+\zeta}{\eta+\theta-1}\right]^{\zeta} \int_{a}^{\infty} \frac{z(t) h^{\zeta}(t)\left[\bar{H}_{2}^{\rho}(t)\right]^{\eta}}{\left[G_{2}^{\rho}(t)\right]^{\eta+\theta-\zeta}} \nabla t
$$

which are exactly the same as the results of Theorem 1.3 and Theorem 1.7, respectively. Therefore the result in (ii) is the diamond alpha unification of the delta Hardy-Copson inequality (1.19) and the nabla Hardy-Copson inequality (1.27).

Remark 3.10. Although inequality (3.8) is better than inequality (3.9), it is worth mentioning inequality (3.9) due to the fact that the mixed integrals disappear and there exist only diamond alpha integrals on the both sides of the inequality (3.9). 
The next theorem provides diamond alpha unifications of the previous Hardy-Copson type inequalities given for $\zeta>1, \eta \geq 0$ and $\eta+\theta>1$. These previous Hardy-Copson type inequalities are listed as follows:

(a) The discrete inequality (1.1) obtained by Hardy as inequality (2) in [28, Theorem B], the discrete inequality (1.4) obtained by Copson [20, Theorem 1.1] and the discrete inequality (1.7) obtained by Bennett [11, Corollary 3] or Leindler [45, Proposition 1].

(b) The continuous inequality (1.2) obtained by Hardy as inequality (4) in [28, Theorem B], the continuous inequality (1.3) obtained by Hardy et al. [30, Theorem 330], the continuous inequality (1.10) obtained by Copson [21, Theorem 1] and the continuous inequality (1.12) obtained by Pachpatte as inequality (6) in [54, Theorem 1] and Pečarić and Hanjš as inequality (3) in [55, Theorem 1].

(c) The delta inequality (1.21) obtained by Kayar and Kaymakçalan [33, Remark 3.2] and Saker et al. [64].

(d) The nabla inequality (1.29) obtained by Kayar and Kaymakçalan [33, Theorem 3.1].

Theorem 3.11. Let the fuctions $\bar{G}_{1}, \bar{G}_{2}, H_{1}, H_{2}, H, \widetilde{H}, \bar{G}$ be defined as in Theorem 3.1 and Theorem 3.4. Let $\frac{\overline{G_{1}}(t)}{\overline{G_{1}^{\sigma}}(t)} \geq \frac{1}{L_{1}}>0$ and $\frac{\overline{G_{2}}(t)}{\overline{G_{2}}(t)} \geq \frac{1}{L_{2}}>0$ and $L=\min \left\{L_{1}, L_{2}\right\}$. If $\zeta>1, \eta \geq 0$ and $\eta+\theta>1$ are real constants, then

(i)

(ii)

$$
\begin{aligned}
\int_{a}^{\infty} \frac{z(t)[H(t)]^{\eta+\zeta}}{[\bar{G}(t)]^{\eta+\theta}} \diamond_{\alpha} t & \leq\left[L_{1}^{\eta+\theta-1} \frac{\eta+\zeta}{\eta+\theta-1}\right]^{\zeta} \int_{a}^{\infty} \frac{z(t) h^{\zeta}(t)\left[H_{1}^{\sigma}(t)\right]^{\eta}}{\left[\bar{G}_{1}^{\sigma}(t)\right]^{\eta+\theta-\zeta}} \Delta t \\
& +\left[L_{2}^{\eta+\theta-1} \frac{\eta+\zeta}{\eta+\theta-1}\right]^{\zeta} \int_{a}^{\infty} \frac{z(t) h^{\zeta}(t)\left[H_{2}(t)\right]^{\eta}}{\left[\bar{G}_{2}(t)\right]^{\eta+\theta-\zeta}} \nabla t
\end{aligned}
$$

$$
\begin{aligned}
\alpha \int_{a}^{\infty} \frac{z(t)\left[H_{1}^{\sigma}(t)\right]^{\eta+\zeta}}{\left[\bar{G}_{1}^{\sigma}(t)\right]^{\eta+\theta}} \Delta t & +(1-\alpha) \int_{a}^{\infty} \frac{z(t)\left[H_{2}(t)\right]^{\eta+\zeta}}{\left[\bar{G}_{2}(t)\right]^{\eta+\theta}} \nabla t \\
& \leq\left[L^{\eta+\theta-1} \frac{\eta+\zeta}{\eta+\theta-1}\right]^{\zeta} \int_{a}^{\infty} \frac{z(t) h^{\zeta}(t)[\widetilde{H}(t)]^{\eta}}{[\bar{G}(t)]^{\eta+\theta-\zeta}} \diamond_{\alpha} t
\end{aligned}
$$

(iii)

$$
\int_{a}^{\infty} \frac{z(t)[H(t)]^{\eta+\zeta}}{[\bar{G}(t)]^{\eta+\theta}} \diamond_{\alpha} t \leq\left[L^{\eta+\theta-1} \frac{\eta+\zeta}{\eta+\theta-1}\right]^{\zeta} \int_{a}^{\infty} \frac{z(t) h^{\zeta}(t)[\widetilde{H}(t)]^{\eta}}{[\bar{G}(t)]^{\eta+\theta-\zeta}} \diamond_{\alpha} t
$$

Proof. If we follow the same procedure of the proof of Theorem 3.1 for the delta inequality (1.21) and the nabla inequality (1.29), we obtain the desired result.

Remark 3.12. If $1<\zeta \leq \eta+\theta$, then $\bar{G}(t)$ can be replaced by $\widetilde{G}(t)=\min _{t \in[a, \infty)}\left\{\bar{G}_{1}^{\sigma}(t), \bar{G}_{2}(t)\right\}$ on the right hand sides of the inequalities (3.10) and (3.11).

Remark 3.13. After expressing the right hand side of the inequality (3.10) in terms of delta and nabla integrals, if we choose $\alpha=1$ and $\alpha=0$ in the resulting inequality, then we obtain

$$
\int_{a}^{\infty} \frac{z(t)\left[H_{1}^{\sigma}(t)\right]^{\eta+\zeta}}{\left[\bar{G}_{1}^{\sigma}(t)\right]^{\eta+\theta}} \Delta t \leq\left[L_{1}^{\eta+\theta-1} \frac{\eta+\zeta}{\eta+\theta-1}\right]^{\zeta} \int_{a}^{\infty} \frac{z(t) h^{\zeta}(t)\left[H_{1}^{\sigma}(t)\right]^{\eta}}{\left[\bar{G}_{1}^{\sigma}(t)\right]^{\eta+\theta-\zeta}} \Delta t
$$

and

$$
\int_{a}^{\infty} \frac{z(t)\left[H_{2}(t)\right]^{\eta+\zeta}}{\left[\bar{G}_{2}(t)\right]^{\eta+\theta}} \nabla t \leq\left[L_{2}^{\eta+\theta-1} \frac{\eta+\zeta}{\eta+\theta-1}\right]^{\zeta} \int_{a}^{\infty} \frac{z(t) h^{\zeta}(t)\left[H_{2}(t)\right]^{\eta}}{\left[\bar{G}_{2}(t)\right]^{\eta+\theta-\zeta}} \nabla t
$$


which are exactly the same as the results of Theorem 1.4 and Theorem 1.8, respectively. Therefore the result in (ii) is the diamond alpha unification of the delta Hardy-Copson inequality (1.21) and the nabla Hardy-Copson inequality (1.29).

Remark 3.14. Although inequality (3.10) is better than inequality (3.11), it is worth mentioning inequality (3.11) due to the fact that the mixed integrals disappear and there exist only diamond alpha integrals on the both sides of the inequality (3.11).

\section{Dynamic diamond alpha inequalities-II}

This section is devoted to derive new integral inequalities, which are different than the ones obtained in Section 3 and are established by using the properties of diamond alpha derivative and integral.

Let $\mathbb{T}$ be a regular time scale and $a \in[0, \infty)_{\mathbb{T}}$. We assume that $z(t)$ and $h(t)$ are non-negative, $\diamond_{\alpha}$-differentiable and locally $\diamond_{\alpha}$-integrable functions defined on $\mathbb{T}$.

The next theorem not only provides diamond alpha unifications of the previous HardyCopson type inequalities given for $\zeta>1, \eta \geq 0$ and $0 \leq \eta+\theta<1$ but also yields novel Hardy-Copson type inequalities for the discrete, continuous, delta, nabla and diamond alpha cases when $\zeta>1, \eta \geq 0$ and $\eta+\theta \leq 0$. This novelty is caused by the condition $\eta+\theta \leq 0$, which has not been considered so far. These previous Hardy-Copson type inequalities are listed as follows:

(a) The discrete inequality (1.8) obtained by Bennett [11, Corollary 5] or Leindler [45, Proposition 3].

(b) The continuous inequality obtained by Saker et al. [64, Corollary 2.2] or Kayar and Kaymakçalan [33, Remark 3.22].

(c) The delta inequalities in Theorem 1.1 obtained by Saker et al. [64, Theorem 2.2].

(d) The nabla inequalities in Theorem 1.5 obtained by Kayar and Kaymakçalan [33, Theorem 3.19].

Theorem 4.1. Let the product $(z h)$ be a nondecreasing function on $[a, \infty)_{\mathbb{T}}$. For $G(t)=$ $\int_{t}^{\infty} z(s) \diamond_{\alpha} s$ and $H(t)=\int_{a}^{t} z(s) h(s) \diamond_{\alpha} s$, assume that there exists $L_{1}>0$ such that $\frac{G(t)}{G^{\rho}(t)} \geq L_{1}$ for $t \in(a, \infty)_{\mathbb{T}}$. Let $\zeta>1, \eta \geq 0$.

(i) If $0 \leq \eta+\theta<1$, then

$$
\begin{aligned}
& \int_{a}^{\infty} \frac{z(t)[H(t)]^{\eta+\zeta}}{\left[G^{\rho}(t)\right]^{\eta+\theta}} \diamond_{\alpha} t \leq \frac{\eta+\zeta}{(1-\eta-\theta)(1-\alpha)} \int_{a}^{\infty} \frac{z(t) h(t)[H(t)]^{\eta+\zeta-1}}{\left[G^{\rho}(t)\right]^{\eta+\theta-1}} \diamond_{\alpha} t \\
& \quad \text { and } \\
& \int_{a}^{\infty} \frac{z(t)[H(t)]^{\eta+\zeta}}{\left[G^{\rho}(t)\right]^{\eta+\theta}} \diamond_{\alpha} t \leq\left[\frac{\eta+\zeta}{(1-\eta-\theta)(1-\alpha)}\right]^{\zeta} \int_{a}^{\infty} \frac{z(t) h^{\zeta}(t)[H(t)]^{\eta}}{\left[G^{\rho}(t)\right]^{\eta+\theta-\zeta}} \diamond_{\alpha} t .
\end{aligned}
$$

(ii) If $\eta+\theta \leq 0$, then

$$
\begin{aligned}
& \int_{a}^{\infty} \frac{z(t)[H(t)]^{\eta+\zeta}}{[G(t)]^{\eta+\theta}} \diamond_{\alpha} t \leq \frac{\eta+\zeta}{(1-\eta-\theta)(1-\alpha)} \int_{a}^{\infty} \frac{z(t) h(t)[H(t)]^{\eta+\zeta-1}}{\left[G^{\rho}(t)\right]^{\eta+\theta-1}} \diamond_{\alpha} t \\
& \text { and } \\
& \int_{a}^{\infty} \frac{z(t)[H(t)]^{\eta+\zeta}}{[G(t)]^{\eta+\theta}} \diamond_{\alpha} t \leq\left[\frac{L_{1}^{\eta+\theta-1}(\eta+\zeta)}{(1-\eta-\theta)(1-\alpha)}\right]^{\zeta} \int_{a}^{\infty} \frac{z(t) h^{\zeta}(t)[H(t)]^{\eta}}{[G(t)]^{\eta+\theta-\zeta}} \diamond_{\alpha} t .
\end{aligned}
$$

Proof. (i) Let us define $u(t)=[H(t)]^{\eta+\zeta}[G(t)]^{1-\eta-\theta}$ for $t \in[a, \infty)$. If we take the nabla derivative of the function $u$ by using formula (2.4), we get

$$
u^{\nabla}(t)=\left[H^{\eta+\zeta}(t)\right]\left[G^{1-\eta-\theta}(t)\right]^{\nabla}+\left[H^{\eta+\zeta}(t)\right]^{\nabla}\left[G^{\rho}(t)\right]^{1-\eta-\theta} .
$$


By utilizing Lemma 2.5, one can obtain

$$
\begin{aligned}
H^{\nabla}(t) & =\left[\int_{a}^{t} z(s) f(s) \diamond_{\alpha} s\right]^{\nabla}=\alpha\left[\int_{a}^{t} z(s) f(s) \Delta s\right]^{\nabla}+(1-\alpha)\left[\int_{a}^{t} z(s) h(s) \nabla s\right]^{\nabla} \\
& =\alpha z^{\rho}(t) h^{\rho}(t)+(1-\alpha) z(t) h(t) \geq 0 .
\end{aligned}
$$

By taking account of (4.6) and by employing the formula (2.5), one can observe for $\eta+\zeta>1$ that

$$
\begin{aligned}
{\left[H^{\eta+\zeta}(t)\right]^{\nabla} } & =(\eta+\zeta) H^{\nabla}(t) \int_{0}^{1}\left[H^{\rho}(t)+w \nu(t) H^{\nabla}(t)\right]^{\eta+\zeta-1} d w \\
& =\int_{0}^{1}(\eta+\zeta)\left[\alpha z^{\rho}(t) h^{\rho}(t)+(1-\alpha) z(t) h(t)\right]\left[(1-w) H^{\rho}(t)+w H(t)\right]^{\eta+\zeta-1} d w \\
& \leq \int_{0}^{1}(\eta+\zeta)\left[\alpha z^{\rho}(t) h^{\rho}(t)+(1-\alpha) z(t) h(t)\right][(1-w) H(t)+w H(t)]^{\eta+\zeta-1} d w \\
& \leq(\eta+\zeta) z(t) h(t)[H(t)]^{\eta+\zeta-1},
\end{aligned}
$$

where $H(\rho(t)) \leq H(t)$ and nondecreasing property of $(z h)$ have been used.

Similarly, by Lemma 2.5 , note that

$$
\begin{aligned}
G^{\nabla}(t) & =\left[\int_{t}^{\infty} z(s) \diamond_{\alpha} s\right]^{\nabla}=\alpha\left[\int_{t}^{\infty} z(s) \Delta s\right]^{\nabla}+(1-\alpha)\left[\int_{t}^{\infty} z(s) \nabla s\right]^{\nabla} \\
& =-\alpha z^{\rho}(t)-(1-\alpha) z(t) \leq 0 .
\end{aligned}
$$

It follows from (4.8) and using the formula (2.5) for $0 \leq \eta+\theta<1$ leads to

$$
\begin{aligned}
{\left[G^{1-\eta-\theta}(t)\right]^{\nabla} } & =\int_{0}^{1} \frac{(1-\eta-\theta)\left[-\alpha z^{\rho}(t)-(1-\alpha) z(t)\right] d w}{\left[(1-w) G^{\rho}(t)+w G(t)\right]^{\eta+\theta}} \\
& \leq-(1-\eta-\theta)(1-\alpha) \frac{z(t)}{\left[G^{\rho}(t)\right]^{\eta+\theta}}
\end{aligned}
$$

where $G(\rho(t)) \geq G(t)$ has been used. Using inequalities (4.7) and (4.9) in equation (4.5) yields

$$
u^{\nabla}(t) \leq \frac{(\eta+\zeta) z(t) h(t)[H(t)]^{\eta+\zeta-1}}{\left[G^{\rho}(t)\right]^{\eta+\theta-1}}-(1-\eta-\theta)(1-\alpha)[H(t)]^{\eta+\zeta} \frac{z(t)}{\left[G^{\rho}(t)\right]^{\eta+\theta}}
$$

or

$$
\begin{aligned}
\int_{a}^{\infty} \frac{z(t)[H(t)]^{\eta+\zeta}}{\left[G^{\rho}(t)\right]^{\eta+\theta}} \diamond_{\alpha} t & \leq \frac{(\eta+\zeta)}{(1-\eta-\theta)(1-\alpha)} \int_{a}^{\infty} \frac{z(t) h(t)[H(t)]^{\eta+\zeta-1}}{\left[G^{\rho}(t)\right]^{\eta+\theta-1}} \diamond_{\alpha} t \\
& -\frac{1}{(1-\eta-\theta)(1-\alpha)} \int_{a}^{\infty} u^{\nabla}(t) \diamond_{\alpha} t
\end{aligned}
$$

The definition of $u$ implies $u(\infty)=u(a)=0$ and by employing Lemma 2.6, we obtain

$$
\begin{aligned}
\int_{a}^{\infty} u^{\nabla}(t) \diamond_{\alpha} t & =\alpha \int_{a}^{\infty} u^{\nabla}(t) \Delta t+(1-\alpha) \int_{a}^{\infty} u^{\nabla}(t) \nabla t \\
& =\alpha[u(\rho(\infty))-u(\rho(a))]+(1-\alpha)[u(\infty)-u(a)] \geq 0,
\end{aligned}
$$

where we have imposed that $(-1)^{\eta+\zeta}=-1$. Therefore we can infer that inequality (4.10) becomes

$\int_{a}^{\infty} \frac{z(t)[H(t)]^{\eta+\zeta}}{\left[G^{\rho}(t)\right]^{\eta+\theta}} \diamond_{\alpha} t \leq \frac{\eta+\zeta}{(1-\eta-\theta)(1-\alpha)} \int_{a}^{\infty} \frac{z(t) h(t)[H(t)]^{\eta+\zeta-1}}{\left[G^{\rho}(t)\right]^{\eta+\theta-1}} \diamond_{\alpha} t$,

which is the desired inequality (4.1). 
Since

$\int_{a}^{\infty} \frac{z(t) h(t)[H(t)]^{\eta+\zeta-1}}{\left[G^{\rho}(t)\right]^{\eta+\theta-1}} \diamond_{\alpha} t=\int_{a}^{\infty} \frac{z^{\frac{1}{\zeta}}(t) h(t)[H(t)]^{\frac{\eta}{\zeta}}}{\left[G^{\rho}(t)\right]^{\frac{\eta+\theta-\zeta}{\zeta}}}\left[\frac{z(t)[H(t)]^{\eta+\zeta}}{\left[G^{\rho}(t)\right]^{\eta+\theta}}\right]^{\frac{\zeta-1}{\zeta}} \diamond_{\alpha} t$,

applying Hölder inequality (2.8) with the constants $\zeta>1$ and $\frac{\zeta}{\zeta-1}>1$ to the right hand side of above equation leads to inequality (4.2).

(ii) If we follow the same procedure of the proof of (i), we arrive inequality (4.8). Using inequality (4.8) and the formula (2.5) for $\eta+\theta \leq 0$ leads to

$$
\begin{aligned}
{\left[G^{1-\eta-\theta}(t)\right]^{\nabla} } & =\int_{0}^{1} \frac{(1-\eta-\theta)\left[-\alpha z^{\rho}(t)-(1-\alpha) z(t)\right] d w}{\left[(1-w) G^{\rho}(t)+w G(t)\right]^{\eta+\theta}} \\
& \leq-(1-\eta-\theta)(1-\alpha) \frac{z(t)}{[G(t)]^{\eta+\theta}}
\end{aligned}
$$

where $G(\rho(t)) \geq G(t)$ has been used. Then continuing in the manner of the proof of (i) by using inequalities (4.7) and (4.12) in equation (4.5) yields

$$
\begin{aligned}
\int_{a}^{\infty} \frac{z(t)[H(t)]^{\eta+\zeta}}{[G(t)]^{\eta+\theta}} \diamond_{\alpha} t & \leq \frac{(\eta+\zeta)}{(1-\eta-\theta)(1-\alpha)} \int_{a}^{\infty} \frac{z(t) h(t)[H(t)]^{\eta+\zeta-1}}{\left[G^{\rho}(t)\right]^{\eta+\theta-1}} \diamond_{\alpha} t \\
& -\frac{1}{(1-\eta-\theta)(1-\alpha)} \int_{a}^{\infty} u^{\nabla}(t) \diamond_{\alpha} t .
\end{aligned}
$$

Then the desired inequality (4.3) can be established by taking account of (4.11). After using $\frac{G(t)}{G^{\rho}(t)} \geq L_{1}$ on the right hand side of inequality (4.3) and applying Hölder inequality (2.8) with the constants $\zeta>1$ and $\frac{\zeta}{\zeta-1}>1$ to the resulting integral, one can obtain inequality (4.4).

Remark 4.2. Special cases of the diamond alpha Hardy-Copson type inequalities (4.1)-(4.4) can be seen below.

(i) After expressing inequality (4.1) and inequality (4.2) in terms of delta and nabla integrals, for $\zeta>1, \eta \geq 0$ and $0 \leq \eta+\theta<1$, choosing $\alpha=0$ in those inequalities yields

$$
\int_{a}^{\infty} \frac{z(t)[H(t)]^{\eta+\zeta}}{\left[G^{\rho}(t)\right]^{\eta+\theta}} \nabla t \leq \frac{\eta+\zeta}{1-\eta-\theta} \int_{a}^{\infty} \frac{z(t) h(t)[H(t)]^{\eta+\zeta-1}}{\left[G^{\rho}(t)\right]^{\eta+\theta-1}} \nabla t
$$

and

$$
\int_{a}^{\infty} \frac{z(t)[H(t)]^{\eta+\zeta}}{\left[G^{\rho}(t)\right]^{\eta+\theta}} \nabla t \leq\left[\frac{\eta+\zeta}{1-\eta-\theta}\right]^{\zeta} \int_{a}^{\infty} \frac{z(t) h^{\zeta}(t)[H(t)]^{\eta}}{\left[G^{\rho}(t)\right]^{\eta+\theta-\zeta}} \nabla t
$$

respectively. Although one of the hypotheses of Theorem 1.5 is $\eta+\theta<1$, in fact, this theorem was proved only for $0 \leq \eta+\theta<1$. Therefore inequality (4.13) and inequality (4.14) coincide with Hardy-Copson type inequalities (1.22) and (1.23), respectively, obtained by nabla time scale calculus. We can conclude that (i) of Theorem 4.1 is a diamond alpha unification of Theorem 1.5.

(ii) After expressing inequality (4.3) and inequality (4.4) in terms of delta and nabla integrals, for $\zeta>1, \eta \geq 0$ and $\eta+\theta \leq 0$, choosing $\alpha=0$ in those inequalities yields

$$
\int_{a}^{\infty} \frac{z(t)[H(t)]^{\eta+\zeta}}{[G(t)]^{\eta+\theta}} \nabla t \leq \frac{\eta+\zeta}{1-\eta-\theta} \int_{a}^{\infty} \frac{z(t) h(t)[H(t)]^{\eta+\zeta-1}}{\left[G^{\rho}(t)\right]^{\eta+\theta-1}} \nabla t
$$


and

$\int_{a}^{\infty} \frac{z(t)[H(t)]^{\eta+\zeta}}{[G(t)]^{\eta+\theta}} \nabla t \leq\left[\frac{L_{1}^{\eta+\theta-1}(\eta+\zeta)}{1-\eta-\theta}\right]^{\zeta} \int_{a}^{\infty} \frac{z(t) h^{\zeta}(t)[H(t)]^{\eta}}{[G(t)]^{\eta+\theta-\zeta}} \nabla t$

respectively. Since the condition $\eta+\theta \leq 0$ has not been considered for any time scale so far, nabla Hardy-Copson type inequalities (4.15) and (4.16) appear in the literature for the first time. Therefore (ii) of Theorem 4.1 is not only a diamond alpha unification of Theorem 1.5 but also provides novel results for diamond alpha and nabla time scale calculi.

The next theorem not only provides diamond alpha unifications of the previous HardyCopson type inequalities given for $\zeta>1, \eta \geq 0$ and $0 \leq \eta+\theta<1$ but also yields novel Hardy-Copson type inequalities for the discrete, continuous, delta, nabla and diamond alpha cases when $\zeta>1, \eta \geq 0$ and $\eta+\theta \leq 0$. This novelty is caused by the condition $\eta+\theta \leq 0$, which has not been considered so far. These previous Hardy-Copson type inequalities are listed as follows:

(a) The discrete inequality (1.5) obtained by Copson [20, Theorem 2.1] and the discrete inequality (1.9) obtained by Bennett [11, Corollary 4] or Leindler [45, Proposition $3]$.

(b) The continuous inequality (1.3) obtained by Hardy et al. [30, Theorem 330], the continuous inequality (1.11) obtained by Copson [21, Theorem 3] and the continuous inequality (1.13) obtained by Pachpatte as inequality (6) in [54, Theorem 1] and Pečarić and Hanjš as inequality (9) in [55, Theorem 3].

(c) The delta inequalities in Theorem 1.2 obtained by Saker et al. [64, Theorem 2.1].

(d) The nabla inequalities in Theorem 1.6 obtained by Kayar and Kaymakçalan [33, Theorem 3.13].

Theorem 4.3. Let the product $(z h)$ be a nonincreasing function on $[a, \infty)_{\mathbb{T}}$. For $\bar{G}(t)=$ $\int_{a}^{t} z(s) \diamond_{\alpha} s$, and $\bar{H}(t)=\int_{t}^{\infty} z(s) h(s) \diamond_{\alpha} s$, assume that there exists $M_{1}>0$ such that $\frac{\bar{G}(t)}{\bar{G}^{\sigma}(t)} \geq M_{1}$ for $t \in(a, \infty)_{\mathbb{T}}$. Let $\zeta>1, \eta \geq 0$.

(i) If $0 \leq \eta+\theta<1$, then

$\int_{a}^{\infty} \frac{z(t)[\bar{H}(t)]^{\eta+\zeta}}{\left[\bar{G}^{\sigma}(t)\right]^{\eta+\theta}} \diamond_{\alpha} t \leq \frac{\eta+\zeta}{\alpha(1-\eta-\theta)} \int_{a}^{\infty} \frac{z(t) h(t)[\bar{H}(t)]^{\eta+\zeta-1}}{\left[\bar{G}^{\sigma}(t)\right]^{\eta+\theta-1}} \diamond_{\alpha} t$

and

$\int_{a}^{\infty} \frac{z(t)[\bar{H}(t)]^{\eta+\zeta}}{\left[\bar{G}^{\sigma}(t)\right]^{\eta+\theta}} \diamond_{\alpha} t \leq\left[\frac{\eta+\zeta}{\alpha(1-\eta-\theta)}\right]^{\zeta} \int_{a}^{\infty} \frac{z(t) h^{\zeta}(t)[\bar{H}(t)]^{\eta}}{\left[\bar{G}^{\sigma}(t)\right]^{\eta+\theta-\zeta}} \diamond_{\alpha} t$.

(ii) If $\eta+\theta \leq 0$, then

$$
\begin{gathered}
\int_{a}^{\infty} \frac{z(t)[\bar{H}(t)]^{\eta+\zeta}}{[\bar{G}(t)]^{\eta+\theta}} \diamond_{\alpha} t \leq \frac{\eta+\zeta}{\alpha(1-\eta-\theta)} \int_{a}^{\infty} \frac{z(t) h(t)[\bar{H}(t)]^{\eta+\zeta-1}}{\left[\bar{G}^{\sigma}(t)\right]^{\eta+\theta-1}} \diamond_{\alpha} t \\
\quad \text { and } \\
\int_{a}^{\infty} \frac{z(t)[\bar{H}(t)]^{\eta+\zeta}}{[\bar{G}(t)]^{\eta+\theta}} \diamond_{\alpha} t \leq\left[\frac{M_{1}^{\eta+\theta-1}(\eta+\zeta)}{\alpha(1-\eta-\theta)}\right]^{\zeta} \int_{a}^{\infty} \frac{z(t) h^{\zeta}(t)[\bar{H}(t)]^{\eta}}{[\bar{G}(t)]^{\eta+\theta-\zeta}} \diamond_{\alpha} t .
\end{gathered}
$$

Proof. (i) Let us define $u(t)=[\bar{H}(t)]^{\eta+\zeta}[\bar{G}(t)]^{1-\eta-\theta}$ for $t \in[a, \infty)$. If we take the delta derivative of the function $u$ by using formula (2.1), we get

$$
u^{\Delta}(t)=\left[\bar{H}^{\eta+\zeta}(t)\right]\left[\bar{G}^{1-\eta-\theta}(t)\right]^{\Delta}+\left[\bar{H}^{\eta+\zeta}(t)\right]^{\Delta}\left[\bar{G}^{\sigma}(t)\right]^{1-\eta-\theta} .
$$


By utilizing Lemma 2.5, one can obtain

$$
\begin{aligned}
\bar{H}^{\Delta}(t) & =\left[\int_{t}^{\infty} z(s) h(s) \diamond_{\alpha} s\right]^{\Delta}=\alpha\left[\int_{t}^{\infty} z(s) h(s) \Delta s\right]^{\Delta}+(1-\alpha)\left[\int_{t}^{\infty} z(s) h(s) \nabla s\right]^{\Delta} \\
& =-\alpha z(t) h(t)-(1-\alpha) z^{\sigma}(t) h^{\sigma}(t) \leq 0 .
\end{aligned}
$$

By taking account of (4.22) and by employing the formula (2.2), one can observe for $\eta+\zeta>1$ that

$$
\begin{aligned}
{\left[\bar{H}^{\eta+\zeta}(t)\right]^{\Delta} } & =(\eta+\zeta) \bar{H}^{\Delta}(t) \bar{H}^{\eta+\zeta-1}(c) \\
& =(\eta+\zeta)\left[-\alpha z(t) h(t)-(1-\alpha) z^{\sigma}(t) h^{\sigma}(t)\right] \bar{H}^{\eta+\zeta-1}(c) \\
& \geq-(\eta+\zeta) z(t) h(t)[\bar{H}(t)]^{\eta+\zeta-1},
\end{aligned}
$$

where $\bar{H}(\sigma(t)) \leq \bar{H}(c) \leq \bar{H}(t)$ and nonincreasing property of $(z h)$ have been used.

Similarly, by Lemma 2.5 , note that

$$
\begin{aligned}
\bar{G}^{\Delta}(t) & =\left[\int_{a}^{t} z(s) \diamond_{\alpha} s\right]^{\Delta}=\alpha\left[\int_{a}^{t} z(s) \Delta s\right]^{\Delta}+(1-\alpha)\left[\int_{a}^{t} z(s) \nabla s\right]^{\Delta} \\
& =\alpha z(t)+(1-\alpha) z^{\sigma}(t) \geq 0 .
\end{aligned}
$$

It follows from (4.24) and using the formula (2.5), for $0 \leq \eta+\theta<1$, leads to

$$
\begin{aligned}
{\left[\bar{G}^{1-\eta-\theta}(t)\right]^{\Delta} } & =(1-\eta-\theta) \bar{G}^{\Delta}(t) \int_{0}^{1}\left[\bar{G}(t)+w \mu(t) \bar{G}^{\Delta}(t)\right]^{-\eta-\theta} d w \\
& =\int_{0}^{1} \frac{(1-\eta-\theta)\left[\alpha z(t)+(1-\alpha) z^{\sigma}(t)\right] d w}{\left[(1-w) \bar{G}(t)+w \bar{G}^{\sigma}(t)\right]^{\eta+\theta}} \\
& \geq \int_{0}^{1} \frac{(1-\eta-\theta) \alpha z(t) d w}{\left[(1-w) \bar{G}^{\sigma}(t)+w \bar{G}^{\sigma}(t)\right]^{\eta+\theta}}=\frac{\alpha(1-\eta-\theta) z(t) d w}{\left[\bar{G}^{\sigma}(t)\right]^{\eta+\theta}},
\end{aligned}
$$

where $\bar{G}(t) \leq \bar{G}(\sigma(t))$ has been used. Using inequalities (4.23) and (4.25) in equation (4.21) yields

$$
u^{\Delta}(t) \geq-(\eta+\zeta) z(t) h(t)[\bar{H}(t)]^{\eta+\zeta-1}\left[\bar{G}^{\sigma}(t)\right]^{1-\eta-\theta}+\frac{\alpha(1-\eta-\theta) z(t)}{\left[\bar{G}^{\sigma}(t)\right]^{\eta+\theta}}[\bar{H}(t)]^{\eta+\zeta}
$$

or

$$
\begin{aligned}
\int_{a}^{\infty} \frac{z(t)[\bar{H}(t)]^{\eta+\zeta}}{\left[\bar{G}^{\sigma}(t)\right]^{\eta+\theta}} \diamond_{\alpha} t & \leq \frac{(\eta+\zeta)}{\alpha(1-\eta-\theta)} \int_{a}^{\infty} \frac{z(t) h(t)[\bar{H}(t)]^{\eta+\zeta-1}}{\left[\bar{G}^{\sigma}(t)\right]^{\eta+\theta-1}} \diamond_{\alpha} t \\
& +\frac{1}{\alpha(1-\eta-\theta)} \int_{a}^{\infty} u^{\Delta}(t) \diamond_{\alpha} t
\end{aligned}
$$

The definition of $u$ implies $u(\infty)=u(a)=0$ and by using Lemma 2.6, we obtain

$$
\begin{aligned}
\int_{a}^{\infty} u^{\Delta}(t) \diamond_{\alpha} t & =\alpha \int_{a}^{\infty} u^{\Delta}(t) \Delta t+(1-\alpha) \int_{a}^{\infty} u^{\Delta}(t) \nabla t \\
& =\alpha[u(\infty)-u(a)]+(1-\alpha)[u(\sigma(\infty))-u(\sigma(a))] \leq 0,
\end{aligned}
$$

where we have imposed that $(-1)^{\eta+\zeta}=-1$.

Therefore we can infer that the inequality (4.26) becomes as

$$
\int_{a}^{\infty} \frac{z(t)[\bar{H}(t)]^{\eta+\zeta}}{\left[\bar{G}^{\sigma}(t)\right]^{\eta+\theta}} \diamond_{\alpha} t \leq \frac{(\eta+\zeta)}{\alpha(1-\eta-\theta)} \int_{a}^{\infty} \frac{z(t) h(t)[\bar{H}(t)]^{\eta+\zeta-1}}{\left[\bar{G}^{\sigma}(t)\right]^{\eta+\theta-1}} \diamond_{\alpha} t,
$$

which is the desired inequality (4.17).

Inequality (4.18) can be obtained by applying Hölder inequality (2.8) with the constants $\zeta>1$ and $\frac{\zeta}{\zeta-1}>1$ to the right hand side of inequality (4.17). 
(ii) If we follow the same procedure of the proof of (i), we arrive inequality (4.24). Using inequality (4.24) and the formula (2.5) for $\eta+\theta \leq 0$ leads to

$$
\left[\bar{G}^{1-\eta-\theta}(t)\right]^{\nabla}=\int_{0}^{1} \frac{(1-\eta-\theta)\left[\alpha z(t)+(1-\alpha) z^{\sigma}(t)\right] d w}{\left[(1-w) \bar{G}(t)+w \bar{G}^{\sigma}(t)\right]^{\eta+\theta}} \geq \alpha(1-\eta-\theta) \frac{z(t)}{[\bar{G}(t)]^{\eta+\theta}},
$$

where $\bar{G}^{\sigma}(t) \geq \bar{G}(t)$ has been used. Then continuing in the manner of the proof of (i) by using inequalities (4.23) and (4.28) in equation (4.21) yields

$$
\begin{aligned}
\int_{a}^{\infty} \frac{z(t)[\bar{H}(t)]^{\eta+\zeta}}{[\bar{G}(t)]^{\eta+\theta}} \diamond_{\alpha} t & \leq \frac{\eta+\zeta}{\alpha(1-\eta-\theta)} \int_{a}^{\infty} \frac{z(t) h(t)[\bar{H}(t)]^{\eta+\zeta-1}}{\left[\bar{G}^{\sigma}(t)\right]^{\eta+\theta-1}} \diamond_{\alpha} t \\
& -\frac{1}{\alpha(1-\eta-\theta)} \int_{a}^{\infty} u^{\Delta}(t) \diamond_{\alpha} t .
\end{aligned}
$$

Hence the desired inequality (4.19) can be established by taking account of (4.27). After using $\frac{\bar{G}(t)}{\bar{G}^{\sigma}(t)} \geq M_{1}$ on the right hand side of inequality (4.19) and applying Hölder inequality $(2.8)$ with the constants $\zeta>1$ and $\frac{\zeta}{\zeta-1}>1$ to the resulting integral, one can obtain inequality (4.20).

Remark 4.4. Special cases of the diamond alpha Hardy-Copson type inequalities (4.17)-(4.20) can be seen below.

(i) After expressing inequality (4.17) and inequality (4.18) in terms of delta and nabla integrals, for $\zeta>1, \eta \geq 0$ and $0 \leq \eta+\theta<1$, choosing $\alpha=1$ in those inequalities yields

$$
\int_{a}^{\infty} \frac{z(t)[\bar{H}(t)]^{\eta+\zeta}}{\left[\bar{G}^{\sigma}(t)\right]^{\eta+\theta}} \Delta t \leq \frac{\eta+\zeta}{1-\eta-\theta} \int_{a}^{\infty} \frac{z(t) h(t)[\bar{H}(t)]^{\eta+\zeta-1}}{\left[\bar{G}^{\sigma}(t)\right]^{\eta+\theta-1}} \Delta t
$$

and

$$
\int_{a}^{\infty} \frac{z(t)[\bar{H}(t)]^{\eta+\zeta}}{\left[\bar{G}^{\sigma}(t)\right]^{\eta+\theta}} \Delta t \leq\left[\frac{\eta+\zeta}{1-\eta-\theta}\right]^{\zeta} \int_{a}^{\infty} \frac{z(t) h^{\zeta}(t)[\bar{H}(t)]^{\eta}}{\left[\bar{G}^{\sigma}(t)\right]^{\eta+\theta-\zeta}} \Delta t
$$

respectively. Although one of the hypotheses of Theorem 1.2 is $\eta+\theta<1$, in fact, this theorem was proved only for $0 \leq \eta+\theta<1$. Therefore inequality (4.29) and inequality (4.30) coincide with Hardy-Copson type inequalities (1.16) and (1.17), respectively, obtained by delta time scale calculus. We can conclude that (i) of Theorem 4.3 is a diamond alpha unification of Theorem 1.2.

(ii) After expressing inequality (4.19) and inequality (4.20) in terms of delta and nabla integrals, for $\zeta>1, \eta \geq 0$ and $\eta+\theta \leq 0$, choosing $\alpha=1$ in those inequalities yields

$$
\int_{a}^{\infty} \frac{z(t)[\bar{H}(t)]^{\eta+\zeta}}{[\bar{G}(t)]^{\eta+\theta}} \Delta t \leq \frac{\eta+\zeta}{1-\eta-\theta} \int_{a}^{\infty} \frac{z(t) h(t)[\bar{H}(t)]^{\eta+\zeta-1}}{\left[\bar{G}^{\sigma}(t)\right]^{\eta+\theta-1}} \Delta t
$$

and

$$
\int_{a}^{\infty} \frac{z(t)[\bar{H}(t)]^{\eta+\zeta}}{[\bar{G}(t)]^{\eta+\theta}} \Delta t \leq\left[\frac{M_{1}^{\eta+\theta-1}(\eta+\zeta)}{1-\eta-\theta}\right]^{\zeta} \int_{a}^{\infty} \frac{z(t) h^{\zeta}(t)[\bar{H}(t)]^{\eta}}{[\bar{G}(t)]^{\eta+\theta-\zeta}} \Delta t
$$

respectively. Since the condition $\eta+\theta \leq 0$ has not been considered for any time scale so far, delta Hardy-Copson type inequalities (4.31) and (4.32) appear in the literature for the first time. Therefore (ii) of Theorem 4.3 is not only a diamond alpha unification of Theorem 1.2 but also provides novel results for diamond alpha and nabla time scale calculi. 
The next theorem provides diamond alpha unifications of the previous Hardy-Copson type inequalities given for $\zeta>1, \eta \geq 0$ and $\eta+\theta>1$. These previous Hardy-Copson type inequalities are listed as follows:

(a) The discrete inequality (1.1) obtained by Hardy as inequality (2) in [28, Theorem B], the discrete inequality (1.4) obtained by Copson [20, Theorem 1.1] and the discrete inequality (1.7) obtained by Bennett [11, Corollary 3] or Leindler [45, Proposition 1].

(b) The continuous inequality (1.2) obtained by Hardy as inequality (4) in [28, Theorem B], the continuous inequality (1.3) obtained by Hardy et al. [30, Theorem 330], the continuous inequality (1.10) obtained by Copson [21, Theorem 1] and the continuous inequality (1.12) obtained by Pachpatte as inequality (6) in [54, Theorem 1] and Pečarić and Hanjš as inequality (3) in [55, Theorem 1].

(c) The delta inequalities in Theorem 1.4 obtained by Kayar and Kaymakçalan [33, Remark 3.2] and Saker et al. [64].

(d) The nabla inequalities in Theorem 1.8 obtained by Kayar and Kaymakçalan [33, Theorem 3.1].

Theorem 4.5. Let the product $(z h)$ be a nondecreasing function on $[a, \infty)_{\mathbb{T}}$. For $\bar{G}(t)=$ $\int_{a}^{t} z(s) \diamond_{\alpha} s$ and $H(t)=\int_{a}^{t} z(s) h(s) \diamond_{\alpha} s$, assume that there exists $L_{2}>0$ such that $\frac{\bar{G}(t)}{\bar{G}^{\rho}(t)} \leq L_{2}$ for $t \in(a, \infty)_{\mathbb{T}}$. If $\zeta>1, \eta \geq 0$ and $\eta+\theta>1$, then

$$
\int_{a}^{\infty} \frac{z(t)[H(t)]^{\eta+\zeta}}{[\bar{G}(t)]^{\eta+\theta}} \diamond_{\alpha} t \leq \frac{\eta+\zeta}{(1-\alpha)(\eta+\theta-1)} \int_{a}^{\infty} \frac{z(t) h(t)[H(t)]^{\eta+\zeta-1}}{\left[\bar{G}^{\rho}(t)\right]^{\eta+\theta-1}} \diamond_{\alpha} t
$$

and

$$
\int_{a}^{\infty} \frac{z(t)[H(t)]^{\eta+\zeta}}{[\bar{G}(t)]^{\eta+\theta}} \diamond_{\alpha} t \leq\left[\frac{L_{2}^{\eta+\theta-1}(\eta+\zeta)}{(1-\alpha)(\eta+\theta-1)}\right]^{\zeta} \int_{a}^{\infty} \frac{z(t) h^{\zeta}(t)[H(t)]^{\eta}}{[\bar{G}(t)]^{\eta+\theta-\zeta}} \diamond_{\alpha} t .
$$

Proof. Let us define $u(t)=[H(t)]^{\eta+\zeta}[\bar{G}(t)]^{1-\eta-\theta}$ for $t \in[a, \infty)$. If we take the nabla derivative of the function $u$ by using formula (2.4), we get

$$
u^{\nabla}(t)=\left[H^{\eta+\zeta}(t)\right]\left[\bar{G}^{1-\eta-\theta}(t)\right]^{\nabla}+\left[H^{\eta+\zeta}(t)\right]^{\nabla}\left[\bar{G}^{\rho}(t)\right]^{1-\eta-\theta} .
$$

Lemma 2.5 implies that

$$
\begin{aligned}
\bar{G}^{\nabla}(t) & =\left[\int_{a}^{t} z(s) \diamond_{\alpha} s\right]^{\nabla}=\alpha\left[\int_{a}^{t} z(s) \Delta s\right]^{\nabla}+(1-\alpha)\left[\int_{a}^{t} z(s) \nabla s\right]^{\nabla} \\
& =\alpha z^{\rho}(t)+(1-\alpha) z(t) \leq 0 .
\end{aligned}
$$

It follows from (4.36) and using the formula (2.5), for $\eta+\theta>1$, we get

$$
\begin{aligned}
{\left[\bar{G}^{1-\eta-\theta}(t)\right]^{\nabla} } & =\int_{0}^{1} \frac{(1-\eta-\theta) \bar{G}^{\nabla}(t) d w}{\left[\bar{G}^{\rho}(t)+w\left\{\bar{G}(t)-\bar{G}^{\rho}(t)\right\}\right]^{\eta+\theta}} \\
& =\int_{0}^{1} \frac{-(\eta+\theta-1)\left[\alpha z^{\rho}(t)+(1-\alpha) z(t)\right] d w}{\left[(1-w) \bar{G}^{\rho}(t)+w \bar{G}(t)\right]^{\eta+\theta}} \leq \frac{-(\eta+\theta-1)(1-\alpha) z(t)}{[\bar{G}(t)]^{\eta+\theta}}
\end{aligned}
$$

where $\bar{G}(\rho(t)) \leq \bar{G}(t)$ has been used. Using inequalities (4.7) and (4.37) in equation (4.35) yields

$$
u^{\nabla}(t) \leq(\eta+\zeta) z(t) h(t)[H(t)]^{\eta+\zeta-1}\left[\bar{G}^{\rho}(t)\right]^{1-(\eta+\theta)}-\frac{(\eta+\theta-1)(1-\alpha) z(t)[H(t)]^{\eta+\zeta}}{[\bar{G}(t)]^{\eta+\theta}}
$$


or

$$
\begin{aligned}
\int_{a}^{\infty} \frac{z(t)[H(t)]^{\eta+\zeta}}{[\bar{G}(t)]^{\eta+\theta}} \diamond_{\alpha} t & \leq \frac{(\eta+\zeta)}{(\eta+\theta-1)(1-\alpha)} \int_{a}^{\infty} \frac{z(t) h(t)[H(t)]^{\eta+\zeta-1}}{\left[\bar{G}^{\rho}(t)\right]^{\eta+\theta-1}} \diamond_{\alpha} t \\
& -\frac{1}{(\eta+\theta-1)(1-\alpha)} \int_{a}^{\infty} u^{\nabla}(t) \diamond_{\alpha} t .
\end{aligned}
$$

The definition of $u$ implies $u(a)=0$ and by using Lemma 2.6, we obtain

$$
\begin{aligned}
\int_{a}^{\infty} u^{\nabla}(t) \diamond_{\alpha} t & =\alpha \int_{a}^{\infty} u^{\nabla}(t) \Delta t+(1-\alpha) \int_{a}^{\infty} u^{\nabla}(t) \nabla t \\
& =\alpha[u(\rho(\infty))-u(\rho(a))]+(1-\alpha)[u(\infty)-u(a)] \geq 0,
\end{aligned}
$$

where we have imposed that $(-1)^{\zeta-\theta}=-1$. Therefore we can infer that the inequality (4.38) becomes as

$$
\int_{a}^{\infty} \frac{z(t)[H(t)]^{\eta+\zeta}}{[\bar{G}(t)]^{\eta+\theta}} \diamond_{\alpha} t \leq \frac{(\eta+\zeta)}{(\eta+\theta-1)(1-\alpha)} \int_{a}^{\infty} \frac{z(t) h(t)[H(t)]^{\eta+\zeta-1}}{\left[\bar{G}^{\rho}(t)\right]^{\eta+\theta-1}} \diamond_{\alpha} t
$$

which is the desired inequality (4.33).

After using $\frac{\bar{G}(t)}{\bar{G}^{\rho}(t)} \geq L_{2}$ on the right hand side of inequality (4.33) and applying Hölder inequality (2.8) with the constants $\zeta>1$ and $\frac{\zeta}{\zeta-1}>1$ to the resulting integral, one can obtain inequality (4.34).

Remark 4.6. After expressing inequality (4.33) and inequality (4.34) in terms of delta and nabla integrals, for $\zeta>1, \eta \geq 0$ and $\eta+\theta>1$, choosing $\alpha=0$ in those inequalities yields

$$
\int_{a}^{\infty} \frac{z(t)[H(t)]^{\eta+\zeta}}{[\bar{G}(t)]^{\eta+\theta}} \nabla t \leq \frac{\eta+\zeta}{\eta+\theta-1} \int_{a}^{\infty} \frac{z(t) h(t)[H(t)]^{\eta+\zeta-1}}{\left[\bar{G}^{\rho}(t)\right]^{\eta+\theta-1}} \nabla t
$$

and

$$
\int_{a}^{\infty} \frac{z(t)[H(t)]^{\eta+\zeta}}{[\bar{G}(t)]^{\eta+\theta}} \nabla t \leq\left[\frac{L_{2}^{\eta+\theta-1}(\eta+\zeta)}{\eta+\theta-1}\right]^{\zeta} \int_{a}^{\infty} \frac{z(t) h^{\zeta}(t)[H(t)]^{\eta}}{[\bar{G}(t)]^{\eta+\theta-\zeta}} \nabla t,
$$

respectively. Inequality (4.39) and inequality (4.40) coincide with Hardy-Copson type inequalities (1.28) and (1.29), respectively, obtained by nabla time scale calculus. Therefore Theorem 4.5 is a diamond alpha unification of Theorem 1.8.

The next theorem provides diamond alpha unifications of the previous Hardy-Copson type inequalities given for $\zeta>1, \eta \geq 0$ and $\eta+\theta>1$. These previous Hardy-Copson type inequalities are listed as follows:

(a) The discrete inequality (1.6) obtained by Bennett [11, Corollary 6] or Leindler [45, Proposition 4].

(b) The continuous inequality obtained by Saker et al. [64, Corollary 2.3] and by Kayar and Kaymakçalan [33, Remark 3.11].

(c) The delta inequalities in Theorem 1.3 obtained by Saker et al. [64, Theorem 2.3].

(d) The nabla inequalities in Theorem 1.7 obtained by Kayar and Kaymakçalan [33, Theorem 3.8].

Theorem 4.7. Let the product $z h$ be a nonincreasing function on $[a, \infty)_{\mathbb{T}}$. For $G(t)=$ $\int_{t}^{\infty} z(s) \diamond_{\alpha} s$, and $\bar{H}(t)=\int_{t}^{\infty} z(s) h(s) \diamond_{\alpha} s$, assume that there exists $M_{2}>0$ such that $\frac{G(t)}{G^{\sigma}(t)} \leq M_{2}$ for $t \in(a, \infty)_{\mathbb{T}}$. If $\zeta>1, \eta \geq 0$ and $\eta+\theta>1$, then

$$
\int_{a}^{\infty} \frac{z(t)[\bar{H}(t)]^{\eta+\zeta}}{[G(t)]^{\eta+\theta}} \diamond_{\alpha} t \leq \frac{\eta+\zeta}{\alpha(\eta+\theta-1)} \int_{a}^{\infty} \frac{z(t) h(t)[\bar{H}(t)]^{\eta+\zeta-1}}{\left[G^{\sigma}(t)\right]^{\eta+\theta-1}} \diamond_{\alpha} t
$$


and

$$
\int_{a}^{\infty} \frac{z(t)[\bar{H}(t)]^{\eta+\zeta}}{[G(t)]^{\eta+\theta}} \diamond_{\alpha} t \leq\left[\frac{M_{2}^{\eta+\theta-1}(\eta+\zeta)}{\alpha(\eta+\theta-1)}\right]^{\zeta} \int_{a}^{\infty} \frac{z(t) h^{\zeta}(t)[\bar{H}(t)]^{\eta}}{[G(t)]^{\eta+\theta-\zeta}} \diamond_{\alpha} t .
$$

Proof. Let us define $u(t)=[\bar{H}(t)]^{\eta+\zeta}[G(t)]^{1-\eta-\theta}$ for $t \in[a, \infty)$. If we take the delta derivative of the function $u$ by using formula (2.1), we get

$$
u^{\Delta}(t)=\left[\bar{H}^{\eta+\zeta}(t)\right]\left[G^{1-\eta-\theta}(t)\right]^{\Delta}+\left[\bar{H}^{\eta+\zeta}(t)\right]^{\Delta}\left[G^{\sigma}(t)\right]^{1-\eta-\theta} .
$$

By Lemma 2.5, note that

$$
\begin{aligned}
G^{\Delta}(t) & =\left[\int_{t}^{\infty} z(s) \diamond_{\alpha} s\right]^{\Delta}=\alpha\left[\int_{t}^{\infty} z(s) \Delta s\right]^{\Delta}+(1-\alpha)\left[\int_{t}^{\infty} z(s) \nabla s\right]^{\Delta} \\
& =-\alpha z(t)-(1-\alpha) z^{\sigma}(t) \leq 0 .
\end{aligned}
$$

It follows from (4.44) and the formula (2.5), for $\eta+\theta>1$, that

$$
\begin{aligned}
{\left[G^{1-\eta-\theta}(t)\right]^{\Delta} } & =\int_{0}^{1} \frac{(1-\eta-\theta) G^{\Delta}(t) d w}{\left[G(t)+w\left\{G^{\sigma}(t)-G(t)\right\}\right]^{\eta+\theta}} \\
& =\int_{0}^{1} \frac{-(\eta+\theta-1)\left[-\alpha z(t)-(1-\alpha) z^{\sigma}(t)\right] d w}{\left[(1-w) G(t)+w G^{\sigma}(t)\right]^{\eta+\theta}} \geq \frac{\alpha(\eta+\theta-1) z(t)}{[G(t)]^{\eta+\theta}},
\end{aligned}
$$

where $G(t) \geq G(\sigma(t))$ has been used. Using inequalities (4.23) and (4.45) in equation (4.43) yields

or

$$
u^{\Delta}(t) \geq-(\eta+\zeta) z(t) h(t)[\bar{H}(t)]^{\eta+\zeta-1}\left[G^{\sigma}(t)\right]^{1-\eta-\theta}+\frac{\alpha(\eta+\theta-1) z(t)}{[G(t)]^{\eta+\theta}}[\bar{H}(t)]^{\eta+\zeta}
$$

$$
\begin{aligned}
\int_{a}^{\infty} \frac{z(t)[\bar{H}(t)]^{\eta+\zeta}}{[G(t)]^{\eta+\theta}} \diamond_{\alpha} t & \leq \frac{\eta+\zeta}{\alpha(\eta+\theta-1)} \int_{a}^{\infty} \frac{z(t) h(t)[\bar{H}(t)]^{\eta+\zeta-1}}{\left[G^{\sigma}(t)\right]^{\eta+\theta-1}} \diamond_{\alpha} t \\
& +\frac{1}{\alpha(\eta+\theta-1)} \int_{a}^{\infty} u^{\Delta}(t) \diamond_{\alpha} t .
\end{aligned}
$$

The definition of $u$ implies $u(\infty)=0$ and by using Lemma 2.6, we obtain

$$
\begin{aligned}
\int_{a}^{\infty} u^{\Delta}(t) \diamond_{\alpha} t & =\alpha \int_{a}^{\infty} u^{\Delta}(t) \Delta t+(1-\alpha) \int_{a}^{\infty} u^{\Delta}(t) \nabla t \\
& =\alpha[u(\infty)-u(a)]+(1-\alpha)[u(\sigma(\infty))-u(\sigma(a))] \leq 0,
\end{aligned}
$$

where we have imposed that $(-1)^{\zeta-\theta}=1$. Therefore we can infer that the inequality (4.46) becomes as

$$
\int_{a}^{\infty} \frac{z(t)[\bar{H}(t)]^{\eta+\zeta}}{[G(t)]^{\eta+\theta}} \diamond_{\alpha} t \leq \frac{\eta+\zeta}{\alpha(\eta+\theta-1)} \int_{a}^{\infty} \frac{z(t) h(t)[\bar{H}(t)]^{\eta+\zeta-1}}{\left[G^{\sigma}(t)\right]^{\eta+\theta-1}} \diamond_{\alpha} t
$$

which is the desired inequality (4.41).

After using $\frac{G(t)}{G^{\sigma}(t)} \leq M_{2}$ on the right hand side of inequality (4.41) and applying Hölder inequality (2.8) with the constants $\zeta>1$ and $\frac{\zeta}{\zeta-1}>1$ to the resulting integral, one can obtain inequality (4.42).

Remark 4.8. After expressing inequality (4.41) and inequality (4.42) in terms of delta and nabla integrals, for $\zeta>1, \eta \geq 0$ and $\eta+\theta>1$, choosing $\alpha=1$ in those inequalities yields

$$
\int_{a}^{\infty} \frac{z(t)[\bar{H}(t)]^{\eta+\zeta}}{[G(t)]^{\eta+\theta}} \Delta t \leq \frac{\eta+\zeta}{\eta+\theta-1} \int_{a}^{\infty} \frac{z(t) h(t)[\bar{H}(t)]^{\eta+\zeta-1}}{\left[G^{\sigma}(t)\right]^{\eta+\theta-1}} \Delta t
$$


and

$$
\int_{a}^{\infty} \frac{z(t)[\bar{H}(t)]^{\eta+\zeta}}{[G(t)]^{\eta+\theta}} \Delta t \leq\left[\frac{M_{2}^{\eta+\theta-1}(\eta+\zeta)}{\eta+\theta-1}\right]^{\zeta} \int_{a}^{\infty} \frac{z(t) h^{\zeta}(t)[\bar{H}(t)]^{\eta}}{[G(t)]^{\eta+\theta-\zeta}} \Delta t,
$$

respectively. Inequality (4.47) and inequality (4.48) coincide with Hardy-Copson type inequalities (1.18) and (1.19), respectively, obtained by delta time scale calculus. Therefore Theorem 4.7 is a diamond alpha unification of Theorem 1.3.

\section{Conclusion}

Since the time scale calculus enables us to avoid the separate discussion of the two cases, which are continuous and discrete cases, the unification of these cases by the delta and the nabla calculi has gained importance in recent years. However, this unification is not complete unless it is done via diamond alpha calculus which harmonizes the delta and the nabla calculi.

In this paper, diamond alpha Hardy-Copson type inequalities were obtained to unify the results established for the special cases, which are discrete and continuous cases as well as the delta and the nabla approaches. In addition to unification, our results provided new Hardy-Copson type inequalities in the special cases.

We derived two kinds of diamond alpha Hardy-Copson type inequalities. The first kind (mixed type) were based on the fact that diamond alpha integral is the convex linear combination of the delta and the nabla integrals. Since the first type inequalities contained delta, nabla and diamond alpha integrals together, we called them mixed type. The second type inequalities were different from the first type in terms of their forms and their method of proofs. Since the second type inequalities included only diamond alpha integrals, they had more compact forms. Moreover some of the second type inequalities were novel even for the delta and nabla cases.

Another novelty of our paper is the method used in the proofs of the theorems in Section 4. In the existing literature, all the authors employed algebra and algebraic inequalities to obtain diamond alpha integral inequalities. However, the proofs of the present paper were based on the time scale calculus including delta and nabla chain rules. Therefore our new developed method could serve as starting point for the new results in the diamond alpha calculus and for the other diamond alpha integral inequalities.

\section{References}

[1] R. Agarwal, M. Bohner and A. Peterson, Inequalities on time scales: a survey, Math. Inequal. Appl. 4 (4), 535-557, 2001.

[2] R.P. Agarwal, R.R. Mahmoud, S. Saker and C. Tunç, New generalizations of NémethMohapatra type inequalities on time scales, Acta Math. Hungar. 152 (2), 383-403, 2017.

[3] R. Agarwal, D. O'Regan and S. Saker, Dynamic Inequalities on Time Scales, Springer, Cham, 2014.

[4] R. Agarwal, D. O'Regan and S. Saker, Hardy Type Inequalities on Time Scales, Springer, Cham, 2016.

[5] M.R.S. Ammi, R.A.C. Ferreira, and D.F.M. Torres, Diamond-alpha Jensen's inequality on time scales, J. Inequal. Appl. 2008 (Art. ID 576876), 1-13, 2008.

[6] D.R. Anderson, Time-scale integral inequalities, J. Inequal. Pure Appl. Math. 6 (3), Article 66, 1-15, 2005.

[7] N. Atasever, B. Kaymakçalan, G. Lešaja and K. Taş, Generalized diamond-alpha dynamic Opial inequalities, Adv. Difference Equ. 2012 (109), 1-9, 2012.

[8] F.M. Atici and G.S. Guseinov, On Green's functions and positive solutions for boundary value problems on time scales, J. Comput. Appl. Math. 141 (1-2), 75-99, 2002. 
[9] A.A. Balinsky, W.D. Evans and R.T. Lewis, The Analysis and Geometry of Hardy's Inequality, Springer International Publishing, Switzerland, 2015.

[10] P.R. Beesack, Hardy's inequality and its extensions, Pacific J. Math. 11 (1), 39-61, 1961.

[11] G. Bennett, Some elementary inequalities, Quart. J. Math. Oxford Ser. (2) 38 (152), 401-425, 1987.

[12] M. Bohner and O. Duman, Opial-type inequalities for diamond-alpha derivatives and integrals on time scales, Differ. Equ. Dyn. Syst. 18 (1-2), 229237, 2010.

[13] M. Bohner, R. Mahmoud and S.H. Saker, Discrete, continuous, delta, nabla, and diamond-alpha Opial inequalities, Math. Inequal. Appl. 18 (3), 923-940, 2015.

[14] M. Bohner, R.R. Mahmoud and S.H. Saker, Improvements of dynamic Opial-type inequalities and applications, Dynam. Syst. Appl. 24, 229-242, 2015.

[15] M. Bohner and A. Peterson, Dynamic Equations on Time Scales. An Introduction With Applications, Birkhäuser Boston, Inc., Boston, MA, 2001.

[16] M. Bohner and A. Peterson, Advances in Dynamic Equations on Time Scales, Birkhäuser Boston, Inc., Boston, MA, 2003.

[17] M.J. Bohner and S.H. Saker, Sneak-out principle on time scales, J. Math. Inequal. 10 (2), 393403, 2016.

[18] M.J. Bohner and S.H. Saker, Gehring Inequalities on Time Scales, J. Comput. Anal. Appl. 28 (1), 11-23, 2020.

[19] Y.-M. Chu, Q. Xu, and X.-M. Zhang, A note on Hardy's inequality, J. Inequal. Appl. 2014 (271), 1-10, 2014.

[20] E.T. Copson, Note on series of positive terms, J. London Math. Soc. 3 (1), 49-51, 1928.

[21] E.T. Copson, Some integral inequalities, Proc. Roy. Soc. Edinburgh Sect. A 75 (2), 157-164, 1976.

[22] A.A. El-Deeb, H.A. Elsennary and Z.A. Khan, Some reverse inequalities of Hardy type on time scales, Adv. Difference Equ. 2020 (402), 1-18, 2020.

[23] A.A. El-Deeb, H.A. Elsennary and B. Dumitru, Some new Hardy-type inequalities on time scales, Adv. Difference Equ. 2020 (441), 1-22, 2020.

[24] P. Gao and H.Y. Zhao, On Copson's inequalities for $0<p<1$, J. Inequal. Appl. 2020 (72), 1-13, 2020.

[25] G.S. Guseinov and B. Kaymakçalan, Basics of Riemann delta and nabla integration on time scales, J. Difference Equ. Appl. 8 (11), 1001-1017, 2002.

[26] M. Gürses, G.S. Guseinov and B. Silindir, Integrable equations on time scales, J. Math. Phys. 46 (11), 113510, 1-22, 2005.

[27] A.F. Güvenilir, B. Kaymakçalan and N.N. Pelen, Constantin's inequality for nabla and diamond-alpha derivative, J. Inequal. Appl. 2015 (167), 1-17, 2015.

[28] G.H. Hardy, Note on a theorem of Hilbert, Math. Z. 6 (3-4), 314-317, 1920.

[29] G.H. Hardy, Notes on some points in the integral calculus, LX. An inequality between integrals, Messenger Math. 54 (3), 150-156, 1925.

[30] G.H. Hardy, J. E. Littlewood, G. Pólya, Inequalities, Cambridge University Press, London, 1934.

[31] M.M. Iddrisu, A.C. Okpoti and A.K. Gbolagade, Some proofs of the classical integral Hardy inequality, Korean J. Math. 22 (3), 407-417, 2014.

[32] S. Iqbal, M.J.S. Sahir and M. Samraiz, Symmetric Rogers-Hölder's inequalities on diamond-alpha calculus, Int. J. Nonlinear Anal. Appl. 9 (2), 9-19, 2018.

[33] Z. Kayar and B. Kaymakçalan, Hardy-Copson type inequalities for nabla time scale calculus, Turk. J. Math. 45 (2), 1040-1064, 2021.

[34] Z. Kayar and B. Kaymakçalan, Some extended nabla and delta HardyCopson type inequalities with applications in oscillation theory, Bull. Iran. Math. Soc., accepted, doi:10.1007/s41980-021-00651-2. 
[35] Z. Kayar and B. Kaymakçalan, Complements of nabla and delta Hardy-Copson type inequalities and their applications, submitted.

[36] Z. Kayar and B. Kaymakçalan, Extensions of diamond-alpha Hardy-Copson type dynamic inequalities and their applications to oscillation theory, Dyn. Syst. Appl. 30 (7), 1180-1209, 2021.

[37] Z. Kayar and B. Kaymakçalan, Applications of the novel diamond-alpha HardyCopson type dynamic inequalities to half linear difference equations, J. Differ. Equ. Appl., accepted.

[38] Z. Kayar and B. Kaymakçalan, Novel diamond-alpha Bennett-Leindler type dynamic inequalities, submitted.

[39] Z. Kayar, B. Kaymakçalan and N.N. Pelen, Diamond-alpha Bennett-Leindler type dynamic inequalities, Math. Methods Appl. Sci., accepted.

[40] Z. Kayar, B. Kaymakçalan and N.N. Pelen, Bennett-Leindler type inequalities for time scale nabla calculus, Mediterr. J. Math. 18 (14), (2021).

[41] Z. Kayar and B. Kaymakçalan, The complementary nabla Bennett-Leindler type inequalities, Commun. Fac. Sci. Univ. Ank. Ser. A1 Math. Stat., accepted.

[42] A. Kufner, L. Maligranda and L.E. Persson, The Hardy Inequality. About Its History and Some Related Results, Vydavatelský Servis, Pilsen, 2007.

[43] A. Kufner, L.E. Persson and N. Samko, Weighted Inequalities of Hardy Type, World Scientific Publishing Co. Pte. Ltd., Hackensack, NJ, 2017.

[44] P. Lefèvre, A short direct proof of the discrete Hardy inequality, Arch. Math. (Basel). 114 (2), 195-198, 2020.

[45] L. Leindler, Some inequalities pertaining to Bennett's results, Acta Sci. Math. (Szeged). 58 (1-4), 261-279, 1993.

[46] L. Leindler, A Theorem of Hardy-Bennett-Type, Acta Math. Hungar. 78 (4), 315325, 1998.

[47] Z.-W. Liao, Discrete Hardy-type inequalities, Adv. Nonlinear Stud. 15 (4), 805-834, 2015.

[48] A.B. Malinowska and D.F.M. Torres, On the diamond-alpha Riemann integral and mean value theorems on time scales, Dynam. Syst. Appl. 18 (3-4), 469-481, 2009.

[49] N. Masmoudi, About the Hardy Inequality, in: An Invitation to Mathematics. From Competitions to Research, Springer, Heidelberg, 2011.

[50] T.Z. Mirković, Dynamic Opial diamond-alpha integral inequalities involving the power of a function, J. Inequal. Appl. 2017 (139), 1-10, 2017.

[51] D. Mozyrska and D.F.M. Torres, A study of diamond-alpha dynamic equations on regular time scales, Afr. Diaspora J. Math. (N.S.) 8 (1), 35-47, 2009.

[52] E.N. Nikolidakis, A sharp integral Hardy type inequality and applications to Muckenhoupt weights on $\mathbb{R}$, Ann. Acad. Sci. Fenn. Math. 39 (2), 887-896, 2014.

[53] U.M. Özkan, M.Z. Sarikaya and H. Yildirim, Extensions of certain integral inequalities on time scales, Appl. Math. Lett. 21 (10), 993-1000, 2008.

[54] B.G. Pachpatte, On Some Generalizations of Hardys Integral Inequality, J. Math. Anal. Appl. 234 (1), 15-30, 1999.

[55] J. Pečarić and Ž. Hanjš, On some generalizations of inequalities given by B. G. Pachpatte, An. Şttiinţ. Univ. Al. I. Cuza Iaşi. Mat. (N.S.) 45 (1), 103-114, 1999.

[56] N.N. Pelen, Hardy-Sobolev-Mazya inequality for nabla time scale calculus, Eskişehir Technical University Journal of Science and Technology B - Theoretical Sciences 7 (2), 133-145, 2019.

[57] J.W. Rogers Jr. and Q. Sheng, Notes on the diamond-alpha dynamic derivative on time scales, J. Math. Anal. Appl. 326 (1), 228-241, 2007.

[58] S.H. Saker, Dynamic inequalities on time scales: A survey, J. Fractional Calc. \& Appl. 3(S) (2), 1-36, 2012. 
[59] S.H. Saker and R.R. Mahmoud, A connection between weighted Hardy's inequality and half-linear dynamic equations, Adv. Difference Equ. 2014 (129), 1-15, 2019.

[60] S.H. Saker, R.R. Mahmoud and A. Peterson, Some Bennett-Copson type inequalities on time scales, J. Math. Inequal. 10 (2), 471-489, 2016.

[61] S.H. Saker, R.R. Mahmoud, M.M. Osman and R.P. Agarwal, Some new generalized forms of Hardy's type inequality on time scales, Math. Inequal. Appl. 20 (2), 459-481, 2017.

[62] S.H. Saker, D. O'Regan and R.P. Agarwal, Dynamic inequalities of Hardy and Copson type on time scales, Analysis 34 (4), 391-402, 2014.

[63] S.H. Saker, D. O'Regan and R.P. Agarwal, Generalized Hardy, Copson, Leindler and Bennett inequalities on time scales, Math. Nachr. 287 (5-6), 686-698, 2014.

[64] S.H. Saker, M.M. Osman, D. O'Regan and R.P. Agarwal, Inequalities of Hardy type and generalizations on time scales, Analysis 38 (1), 4762, 2018.

[65] S.H. Saker, R.R. Mahmoud and A. Peterson, A unified approach to Copson and Beesack type inequalities on time scales, Math. Inequal. Appl. 21 (4), 985-1002, 2018.

[66] Q. Sheng, M. Fadag, J. Henderson and J.M. Davis, An exploration of combined dynamic derivatives on time scales and their applications, Nonlinear Anal. Real World Appl. 7 (3), 395-413, 2006. 